\title{
Heterogeneity of the GFP fitness landscape and data-driven protein design
}

Louisa Gonzalez Somermeyer ${ }^{1}$, Aubin Fleiss $^{2,3}$, Alexander S. Mishin ${ }^{4}$, Nina G. Bozhanova ${ }^{6}$, Anna A. Igolkina ${ }^{7}$, Jens Meiler ${ }^{6,8}$, Maria-Elisenda Alaball Pujol ${ }^{2,3}$, Ekaterina V. Putintseva ${ }^{5}$, Karen S. Sarkisyan ${ }^{2,3,4^{*}}$, Fyodor A. Kondrashov ${ }^{1 *}$

${ }^{1}$ Institute of Science and Technology Austria, 1 Am Campus, Klosterneuburg, 3400, Austria

${ }^{2}$ Synthetic Biology Group, MRC London Institute of Medical Sciences, London, UK

${ }^{3}$ Institute of Clinical Sciences, Faculty of Medicine, Imperial College London, London, UK

${ }^{4}$ Shemyakin-Ovchinnikov Institute of Bioorganic Chemistry, Russian Academy of Sciences, Moscow, Russia

${ }^{5}$ LabGenius, G.01-06 Cocoa Studios, 100 Drummond Rd, London, SE16 4DG UK

${ }^{6}$ Department of Chemistry, Center for Structural Biology, Vanderbilt University, Nashville, TN

${ }^{7}$ Gregor Mendel Institute, Austrian Academy of Sciences, Vienna BioCenter, 1030 Vienna, Austria

${ }^{8}$ Institute for Drug Discovery, Medical School, Leipzig University, Leipzig, Germany

*Corresponding authors, KSS karen.s.sarkisyan@gmail.com; FAK fvodor.kondrashov@ist.ac.at

Studies of protein fitness landscapes reveal biophysical constraints guiding protein evolution and empower prediction of functional proteins. However, generalisation of these findings is limited due to scarceness of systematic data on fitness landscapes of proteins with a defined evolutionary relationship. We characterized the fitness peaks of four orthologous fluorescent proteins with a broad range of sequence divergence. While two of the four studied fitness peaks were sharp, the other two were considerably flatter, being almost entirely free of epistatic interactions. Counterintuitively, mutationally robust proteins, characterized by a flat fitness peak, were not optimal templates for machine-learning-driven protein design - instead, predictions were more accurate for fragile proteins with epistatic landscapes. Our work paves insights for practical application of fitness landscape heterogeneity in protein engineering.

\section{Introduction}

Understanding the relationship between genotype and phenotype, the fitness landscape, elucidates the fundamental laws of heredity (Canale et al., 2018; de Visser and Krug, 2014; Ferretti et al., 2018; Fragata et al., 2019; Wright, 1932) and may ultimately create novel methods of protein design (Alley et al., 2019; Bryant et al., 2021a; Hirabayashi and Arai, 2019; Wrenbeck et al., 2017; Wu et al., 2019). The fitness landscape is often conceptualised as a multidimensional surface (de Visser and Krug, 2014; Ferretti et al., 2018; Kondrashov and Kondrashov, 2015; Wright, 1932) with one dimension representing fitness, or another phenotype, and the other dimensions each representing a genotype's locus. Originally, the fitness landscape was introduced to describe the relationship between fitness and the entire genome (de Visser and Krug, 2014; Wright, 1932). Over time, the usefulness of the concept of the fitness landscape led to the adaptation of this term to describe the relationship between protein function and its protein-coding gene sequence (Biswas et al., 2021; Ogden et al., 2019; Romero and Arnold, 2009; Wittmann et al., 2021; Zheng et al., 2020). Absolute knowledge of the fitness landscape would reveal the phenotypes conferred by any arbitrary genotype (de Visser and Krug, 2014; Ferretti et al., 2018; Fragata et al., 
2019), with immense and obvious practical implications (Alley et al., 2019; Bryant et al., 2021a; Hirabayashi and Arai, 2019; Kemble et al., 2019; Wrenbeck et al., 2017; Wu et al., 2019). However, sparse experimental data, even for specific genes, and the concomitant lack of understanding of the rules by which fitness landscapes are formed, limit the accuracy of phenotype predictions based on sequence alone (Lässig et al., 2017) [but see (Bryant et al., 2021a; Rocklin et al., 2017; Senior et al., 2020; Wu et al., 2019)].

While several experimentally characterized fitness landscapes for specific proteins have been reported (Hartman and Tullman-Ercek, 2019; Jacquier et al., 2013; Kuo et al., 2020; Melamed et al., 2013; Olson et al., 2014; Sarkisyan et al., 2016), such surveys of large proteins are still hindered by the enormity of the genotype space (de Visser and Krug, 2014; Wright, 1932). Even for the Green Fluorescent Protein (GFP), which is only 250 amino acids long, there are $20^{250}$ possible genotypes. Without complex epistatic interactions between amino acid sites the fitness landscape could be deduced from the independent contribution of each amino acid at each site (Kondrashov and Kondrashov, 2015), requiring just 5000 $(20 * 250)$ measurements of the effects of all single mutations in GFP. However, epistatic interactions between amino acid sites are common (Russ et al., 2020) and many of them are too complex to predict with available data (Pokusaeva et al., 2019). Despite some advances in the development of data-driven approaches to protein design (Biswas et al., 2020, 2018; Bryant et al., 2021a; Kemble et al., 2019), it is still not clear what fraction of the $20^{250}$ sequences of the GFP, or any other gene, must be characterized to approach the coveted absolute knowledge of the fitness landscape (Kemble et al., 2019; Sailer et al., 2020; Zhou and McCandlish, 2020).

Despite lack of data, experiments and theory provide some insights on the global fitness landscape (Fragata et al., 2019; Kemble et al., 2019). Each extant genotype, one that is found in an extant species, is a point of high fitness, or a fitness peak, on the highly dimensional and extraordinarily large genotype space (de Visser and Krug, 2014; Fragata et al., 2019; Maynard Smith, 1970; Wright, 1932). These extant genotypes had a common ancestor, so they must be connected by ridges of high fitness (Gong et al., 2013; Maynard Smith, 1970; Povolotskaya and Kondrashov, 2010). Nevertheless, only an infinitesimally small fraction of all genotypes are functional (fewer than $10^{-11}$ ), those that correspond to fitness peaks and ridges, the rest confer low fitness (Keefe and Szostak, 2001). The fitness peaks are sharp (Bank et al., 2015; Melamed et al., 2013; Sarkisyan et al., 2016) and the ridges are narrow (Gong et al., 2013; Kumar et al., 2017; Pokusaeva et al., 2019; Sailer et al., 2020) and, on average, only a few random mutations in a wildtype sequence reduce its fitness to zero (Hartman and Tullman-Ercek, 2019; Kemble et al., 2019). The sharpness of the peaks is enhanced by negative epistasis, such that a genotype with several random mutations has a lower fitness than expected if mutations acted independently (Haddox et al., 2018; Sarkisyan et al., 2016). Thus, a random walk from a fitness peak eventually leads to an area of the genotype space where only an infinitesimally small fraction of sequences are functional, likely explaining why accurate prediction of functional genotypes at a 
substantial distance away from a functional genotype remains a challenge (Alley et al., 2019; Hirabayashi and Arai, 2019; Russ et al., 2020; Wu et al., 2019).
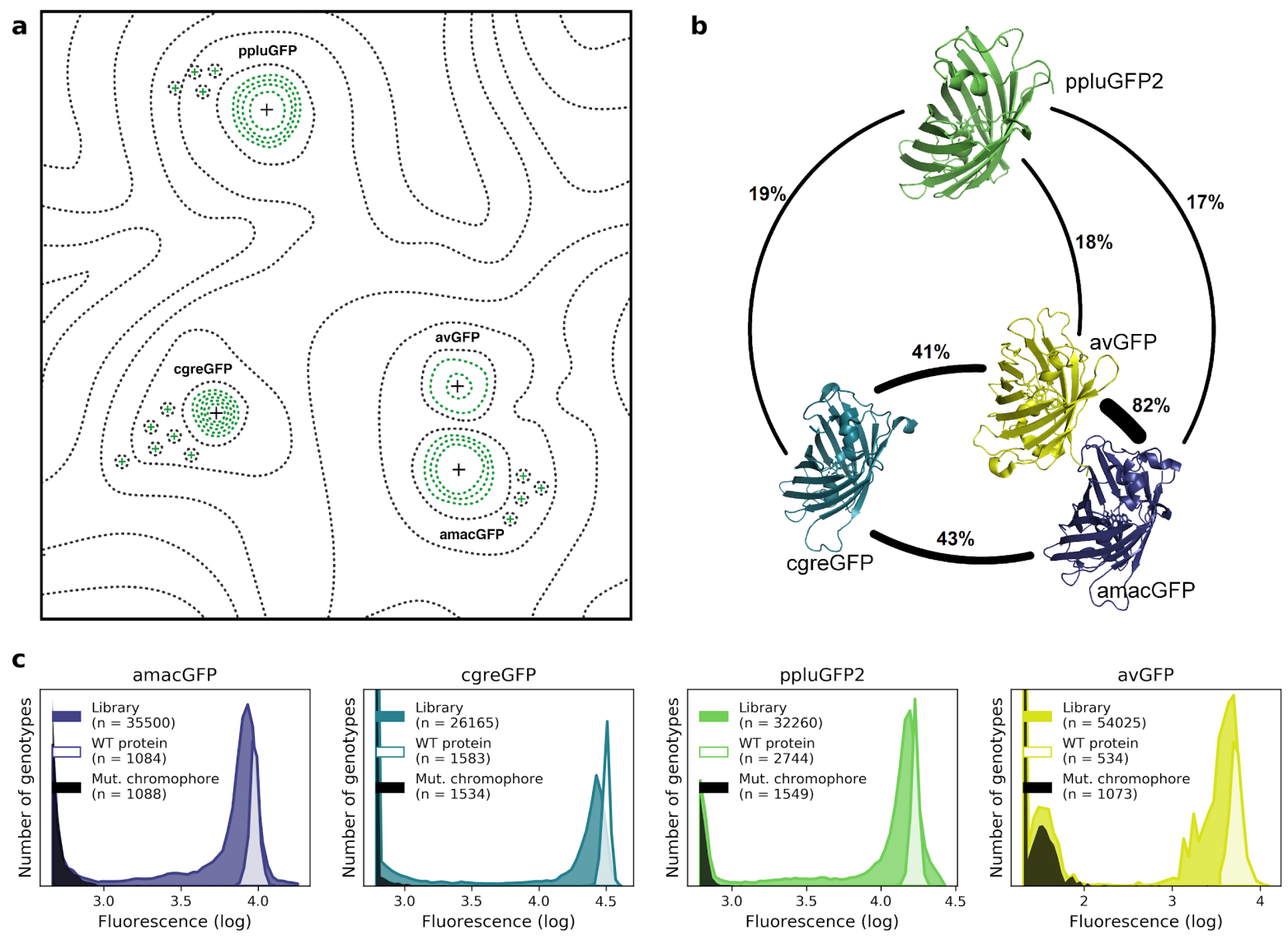

Figure 1. Comparison of four GFP fitness peaks. a, A conceptual representation of the GFP fitness landscape following the visualization proposed by Sewal Wright (Wright, 1932). The black dotted lines represent the unknown regions of the fitness landscape and the green lines the surveyed local fitness peak. Wildtype GFPs (black + ) and predicted functional GFPs $($ green + ) are shown at an approximate scale of sequence divergence from each other. $\mathbf{b}$, Amino acid sequence identity between different orthologs, displayed in percent. c, Distribution of fluorescence of mutant libraries (colour), control wildtype protein sequences (white), and protein sequences containing loss-of-function mutations in the chromophore (black).

The shape of fitness peaks and ridges, and their distribution in genotype space has implications for fundamental questions in evolution (de Visser and Krug, 2014) and practical applications (Sardanyés et al., 2008). Evolution starting at a sharp fitness peak is expected to proceed at a different pace than evolution on a flat one (Bershtein et al., 2006; Codoñer et al., 2006; de Visser et al., 2003; Draghi et al., 2010; Wagner, 2008). Furthermore, it has been suggested that flat fitness peaks, representing robust genotypes, may be 
evolutionarily preferable to sharp peaks, which represent fragile genotypes (Bershtein et al., 2006; de Visser et al., 2003; Draghi et al., 2010; Klug et al., 2019; Zheng et al., 2020). However, how different shapes of fitness peaks may be distributed in genotype space has not been explored (Chan et al., 2017; Kemble et al., 2019). Furthermore, the exploration of the fitness landscape of specific proteins is one of the approaches in protein engineering (Bryant et al., 2021b; Romero and Arnold, 2009; Russ et al., 2020; Wittmann et al., 2021). Such studies explore the fitness landscape of the protein of interest through deep mutational scan of a known protein sequence. Then this information is used to predict novel functional protein sequences that are designed by introducing mutations into the original sequence. Here, we explored the interplay of the heterogeneity of fitness peaks of orthologous sequences and prediction of novel functional protein sequences (Figure 1a). To this end, we compared the fitness peaks of four GFPs that had different levels of sequence divergence from each other. We then used this information to accurately predict novel functional GFPs at considerable sequence divergence to any known GFP sequence.

\section{Results}

To complement the available data on the avGFP fitness peak (Sarkisyan et al., 2016) (GFP from Aequorea victoria, Hydrozoa) we experimentally characterized three additional GFP sequences, each with a different degree of sequence divergence from avGFP: amacGFP (Aequorea macrodactyla, Hydrozoa), cgreGFP (Clytia gregaria, Hydrozoa), and ppluGPF2 (Pontellina plumata, Copepoda), with 18\%, 59\% and 82\% sequence divergence, respectively (Figure 1b; Table 1). For simplicity, we refer to all of these sequences as "wildtype", even though only cgreGFP and ppluGPF2 were identical to the true wildtype sequences, while avGFP and amacGFP contain one and three amino acid substitutions, respectively. amacGFP, ppluGFP2 and cgreGFP were subject to a similar experimental pipeline (Figure S1) as avGFP (Sarkisyan et al., 2016). For each sequence a library of genotypes containing random mutations in the respective GFP sequence was generated by error-prone PCR, in which each GFP gene variant was labelled downstream of its stop codon by a primary barcode, a random combination of nucleotides. This mutant library was expressed as a fusion protein with the red fluorescent protein mKate2 in E. coli cells, which were then sorted based on green fluorescence intensity within a narrow red fluorescence gate, to control for gene expression level and other errors (Figure S2). The DNA barcodes of the sorted cells were sequenced and these data were used to perform a statistical analysis estimating the level of fluorescence of tens of thousands of GFP genotypes. Three notable improvements to the original experimental pipeline were implemented: gene sequence-agnostic library sequencing, genome integration of the construct, and use of secondary barcodes that introduced internal replicas in the experiment (see Methods). These changes resulted in more physiologically relevant expression levels, made the pipeline more scalable, and reduced the variance of fluorescent genotype measurements by a factor of 7 (Figure 1c; Table 1). The new dataset contained 25,000-35,000 genotypes per each of the three additional fitness peaks, with each mutant genotype harboring on average 3-4 mutations relative to its respective wildtype sequence (Table 1). These data, together 
bioRxiv preprint doi: https://doi.org/10.1101/2021.12.08.471728; this version posted December 9, 2021. The copyright holder for this preprint (which was not certified by peer review) is the author/funder, who has granted bioRxiv a license to display the preprint in perpetuity. It is made available under aCC-BY 4.0 International license.

with data from avGFP, were then used in our comparative study of the GFP fitness peaks (Figure 1a).

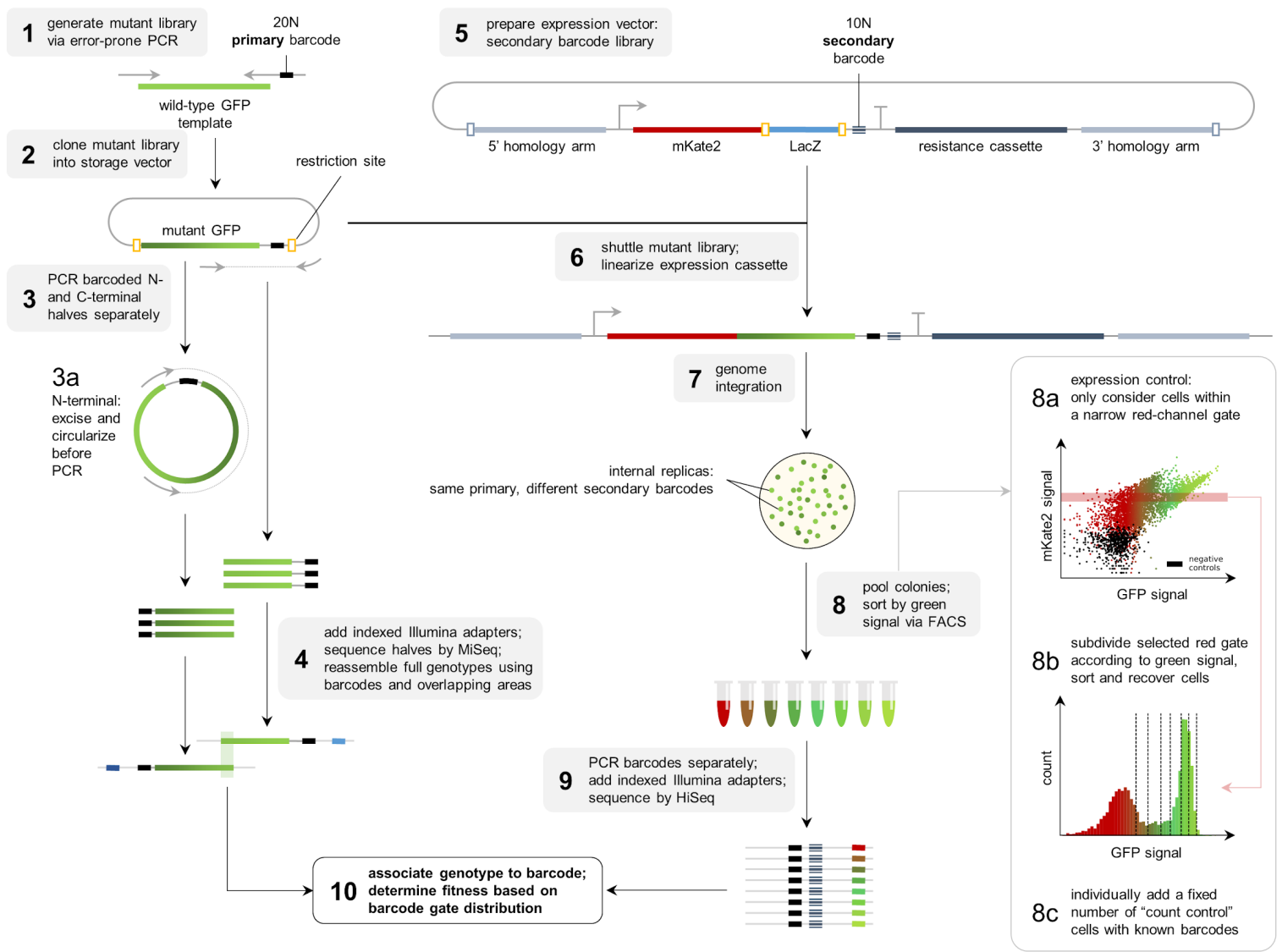

Figure S1. Flowthrough of the experimental methodology. 
Table 1. The dataset in numbers. The avGFP data is from (Sarkisyan et al., 2016).

\begin{tabular}{|c|c|c|c|c|}
\hline Gene & amacGFP & cgreGFP & ppluGFP2 & avGFP \\
\hline $\begin{array}{l}\text { Number of protein genotypes } \\
\text { surveyed }\end{array}$ & 35500 & 26165 & 32260 & 51715 \\
\hline $\begin{array}{l}\text { Average (median) number of AA } \\
\text { substitutions per genotype }\end{array}$ & $4.37(3)$ & $4.23(3)$ & $3.7(2)$ & $3.93(4)$ \\
\hline $\begin{array}{l}\text { Average (median) number of } \\
\text { barcode replicates per protein } \\
\text { genotype }\end{array}$ & $8.7(5)$ & $6.8(5)$ & $12(7)$ & $1.2(1)$ \\
\hline Amino acid identity & $\begin{array}{l}\text { avGFP: } 82 \% \\
\text { cgreGFP: } 43 \% \\
\text { ppluGFP2: } 17 \%\end{array}$ & $\begin{array}{l}\text { avGFP: } 41 \% \\
\text { amacGFP: } 43 \% \\
\text { ppluGFP2: } 19 \%\end{array}$ & $\begin{array}{l}\text { avGFP: } 18 \% \\
\text { amacGFP: } 17 \% \\
\text { cgreGFP: } 19 \%\end{array}$ & $\begin{array}{l}\text { amacGFP: } 82 \% \\
\text { cgreGFP: } 41 \% \\
\text { ppluGFP2: } 18 \%\end{array}$ \\
\hline False positive rate* & $0.55 \%$ (9 of 1635$)$ & $0.75 \%$ (14 of 1860$)$ & $0.49 \%$ (11 of 2242$)$ & $0.24 \%$ (2 of 839$)$ \\
\hline False negative rate* & $0 \%(0$ of 1084$)$ & $0 \%(0$ of 1583$)$ & $0 \%(0$ of 2744$)$ & $0.08 \%$ ( 2 of 2444$)$ \\
\hline $\begin{array}{l}\text { Mean wildtype log10 fluorescence } \\
\text { level } \pm \text { standard deviation }\end{array}$ & $\begin{array}{l}3.97 \pm 0.031 \\
(3.96 \pm 0.030 \text { for } \\
\text { amacGFP:V14L) }\end{array}$ & $4.50 \pm 0.028$ & $4.23 \pm 0.027$ & $3.72 \pm 0.082$ \\
\hline $\begin{array}{l}\text { Fraction of genotypes in which } \\
\text { epistasis cannot be ascertained }\end{array}$ & $7.4 \%$ & $15.9 \%$ & $4.5 \%$ & $16.5 \%$ \\
\hline $\begin{array}{l}\text { Fraction of genotypes displaying } \\
\text { |epistasis } \mid>0.3(>1)^{\star \star *}\end{array}$ & $5.3 \%(0.2 \%)$ & $14.4 \%(5.6 \%)$ & $6.8 \%(0.9 \%)$ & $21.4 \%(11.6 \%)$ \\
\hline $\begin{array}{l}\text { Mutational LD50, loss of } \\
\text { function****}\end{array}$ & $\begin{array}{l}5.8 \\
(5.7 \text { for } \\
\text { amacGFP:V14L) }\end{array}$ & 3.2 & 6.2 & 4.1 \\
\hline $\begin{array}{l}\text { Mutational LD50, loss of } \\
\text { wildtype-level fluorescence } \\
\text { level }\end{array}$ & $\begin{array}{l}1.7 \\
(1.8 \text { for } \\
\text { amacGFP:V14L) }\end{array}$ & 0.9 & 1.7 & 2.2 \\
\hline $\begin{array}{l}\text { Proportion of machine-learning } \\
\text { predicted genotypes displaying } \\
\text { epistasis }<-0.3(<-1)\end{array}$ & $78 \%(46 \%)$ & $57 \%(21 \%)$ & $81 \%(64 \%)$ & NA \\
\hline
\end{tabular}

* False positive rates refer to the fraction of genotypes which are expected to be dark or dim due to chromophore mutations but which were assigned a bright fitness; false negative rates refer to genotypes encoding wildtype protein which were assigned dim or dark fitnesses.

** Calculation of epistasis requires knowledge of a genotype's expected fluorescence, i.e. the sum of contributions of individual mutations. For genotypes with multiple mutations, all individual mutations comprising the genotype must have been measured in isolation.

*** An absolute epistasis value of 0.3 or 1 implies a two-fold or ten-fold difference between the observed and expected fluorescence levels, respectively.

$* * * *$ "Mutational LD50, loss of function" refers to the number of mutations at which $50 \%$ of genotypes are rendered non-functional (i.e. assigned to the darkest FACS gate), obtained by fitting a logistic curve to the fraction of non-functional genotypes at each mutational step (see values in Table S1) and solving for $\mathrm{f}(\mathrm{x})=0.5$; "Mutational LD50, loss of wildtype fluorescence level" refers instead to the number of mutations at which 50\% of genotypes maintain a fluorescence level within two standard deviations of the WT level. 
The four fitness peaks shared substantial similarities (also see (Biswas et al., 2018) for sfGFP). In all cases synonymous variants had no measurable effect on fitness, which may be a consequence of the experimental design aimed to be insensitive to expression levels and, thus, they were pooled for all subsequent analyses (Figure S3a). Mutations in the chromophore eliminated fluorescence (Figure S4a) and mutations of buried amino acid residues had a stronger effect than mutations of residues on the protein surface (Figure $\mathbf{2 b}$; Figure S4b). In all four fitness peaks a threshold effect of accumulating multiple random mutations was found, such that the median level of fluorescence dropped sharply once a certain number of mutations was reached (Figure 2a; Figure S3b; Table S1). The fitness peak shape differed substantially among different GFP sequences. Only 3-4 mutations were necessary for avGFP and cgreGFP, so the corresponding fitness peaks were sharp (Table 1). By contrast, the fitness peaks of amacGFP and ppluGFP2 were substantially flatter, with each tolerating twice as many mutations (Figure 2a; Figure S3b). Furthermore, we characterized the sharpness of the fitness peaks associated with many neighboring sequences, those harbouring a single mutation relative to the wildtype sequence. Fitness peaks of most single-mutation neighbours with high levels of fluorescence were sharper than the respective wildtype fitness peaks (Figure 2c), suggesting a local optimization of robustness of each wildtype sequence (Draghi et al., 2010). Notably, the shape of the wildtype fitness peak showed no straightforward relationship with its respective level of fluorescence (Table 1), as may have been expected (Johnson et al., 2019). 

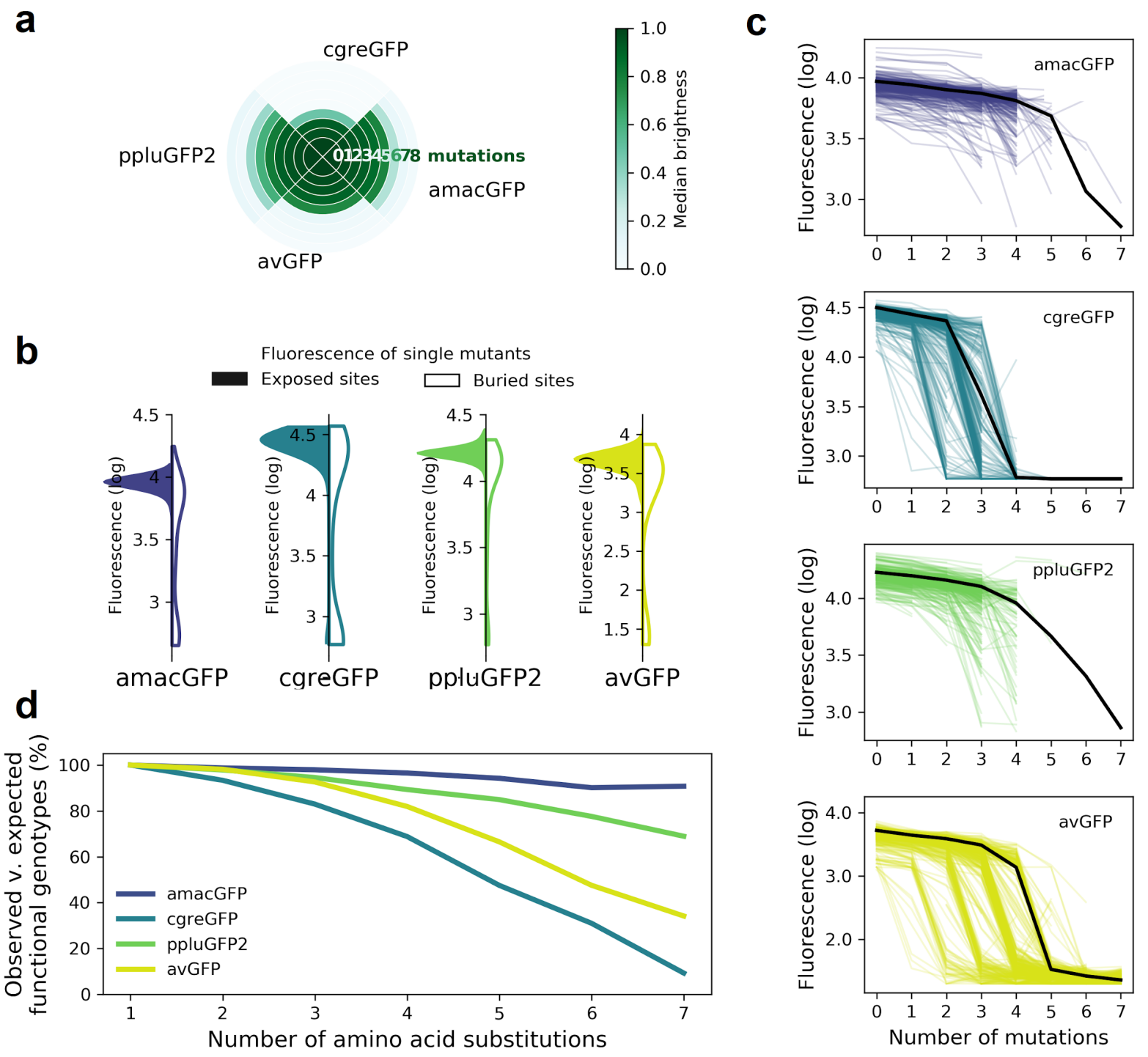

Figure 2. Sequence divergence and epistasis within and between genes. a, The sharpness of different GFP fitness peaks, showing the median relative fluorescence level of genotypes with the corresponding number of mutations away from the wildtype. Fluorescence values are normalized so that the wildtype level equals 1. b. Distribution of fluorescence of genotypes with a single amino acid mutation at exposed (colour) versus buried (white) sites. c, The sharpness of fitness peaks of the genotypes that harbor one mutation relative to the wildtype sequence. Each curve shows the median fluorescence level at various distances away from such genotypes, calculated for points with at least 15 available genotypes. The black lines show the fluorescence level at varying distances from the wildtype sequence. d, The ratio of the number of observed functional genotypes and the number of genotypes expected to be functional under the assumption of no epistatic interactions between amino acid sites; in the absence of epistasis, the expectation is a constant value of $100 \%$. 

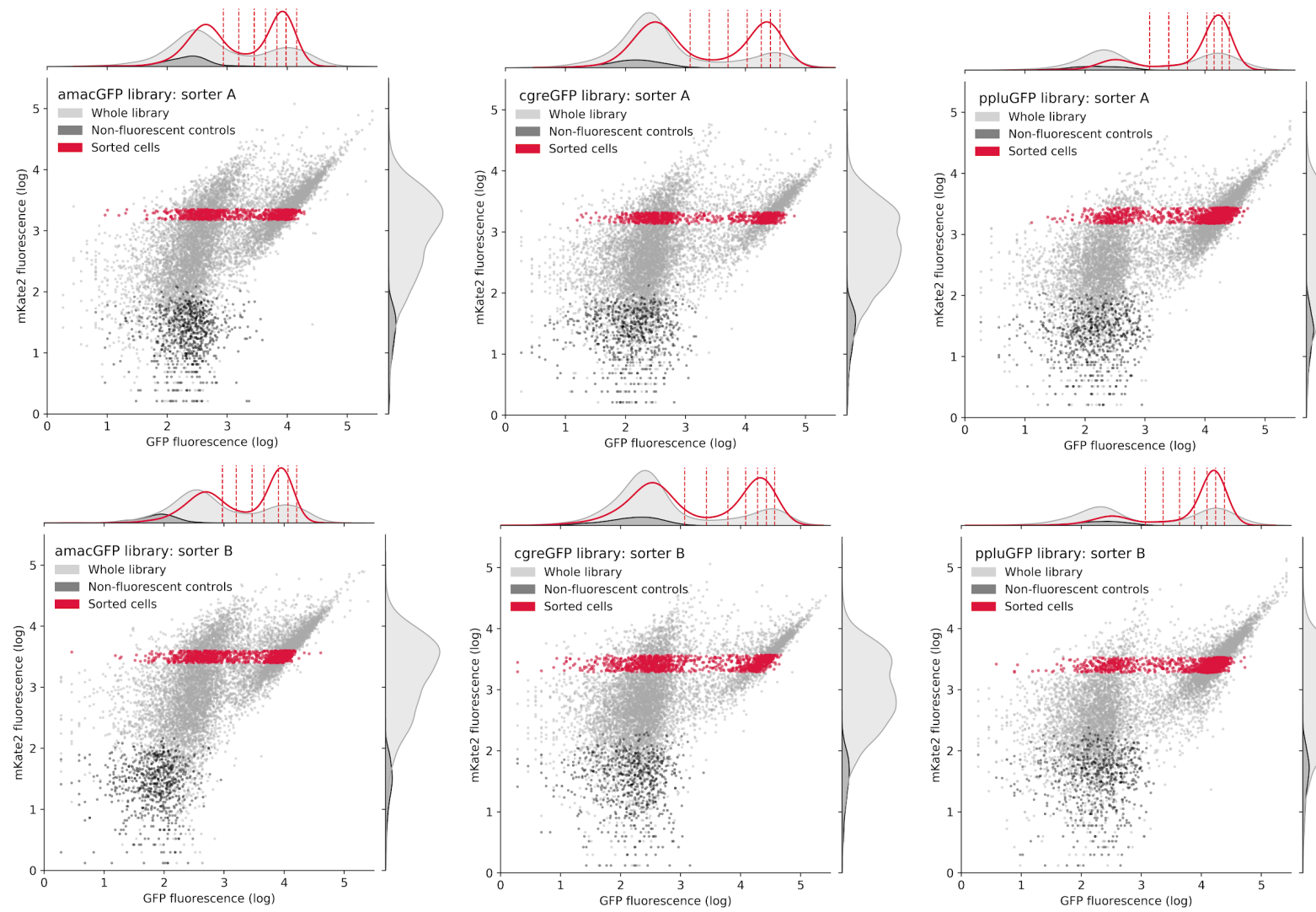

Figure S2. Distribution of cells during FACS sorting. Entire mutant libraries are shown in grey, non-fluorescent negative controls are shown in black. Sorted cells, falling within the selected gate in the mKate2 channel and corresponding to around $10 \%$ of all bacterial cells, are shown in red. Vertical dashed lines in the upper histogram indicate the eight gates in the green channel that cells were recovered from. The histograms indicate the distribution of cells in a single channel.
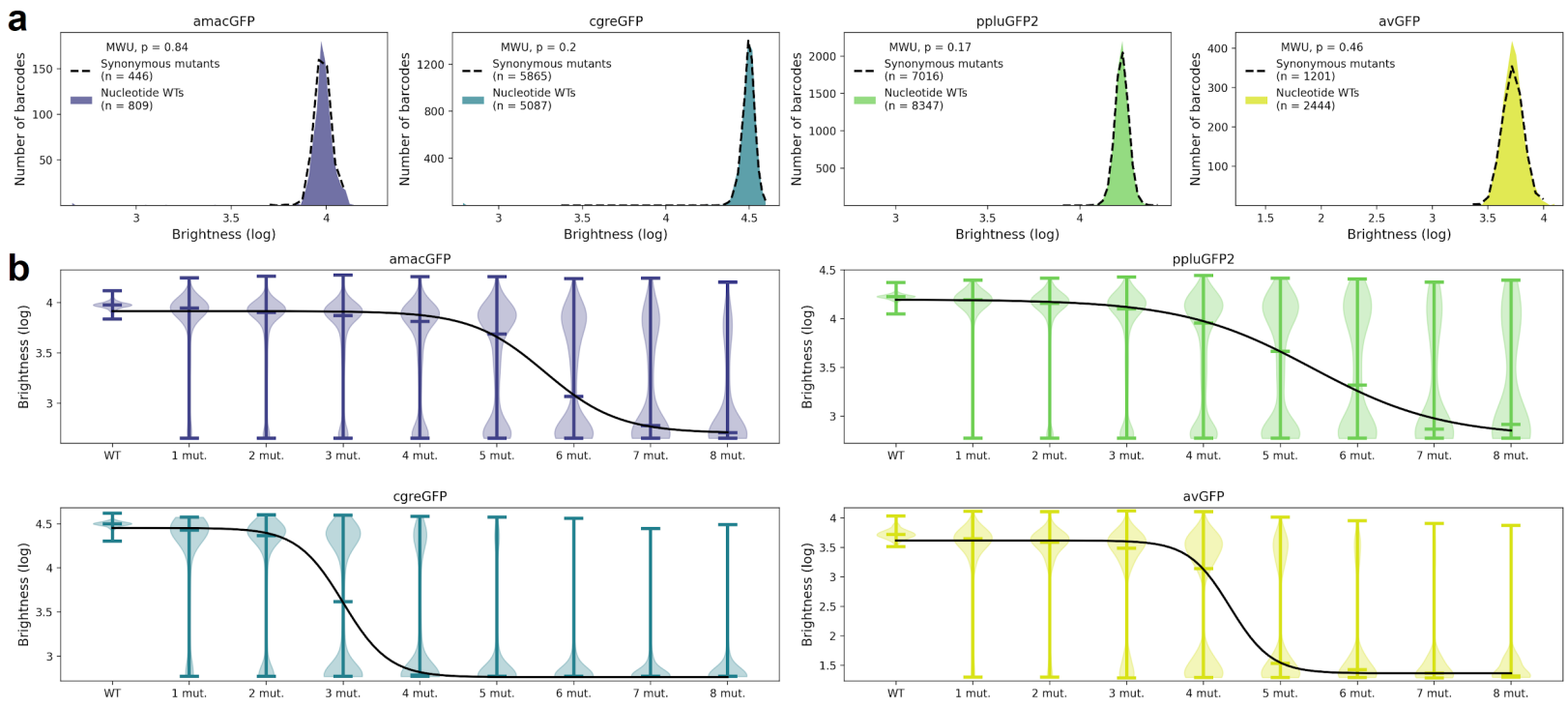
Figure S3. Distributions of libraries and control genotypes. a, Fluorescence level distributions of individual barcodes linked to wild-type nucleotide sequences (colour) versus sequences containing only synonymous mutations (dotted line). The minimum number of observations per barcode was 50. The differences between barcoded wildtypes and synonymous variants in all four libraries was not significant ( $p$-value $>0.17$, two-tailed Mann Whitney U-test). b, Fluorescence level distributions of genotypes at varying distances from the wildtype and the logistic curves fitted to the median fluorescence for each category (black line). 

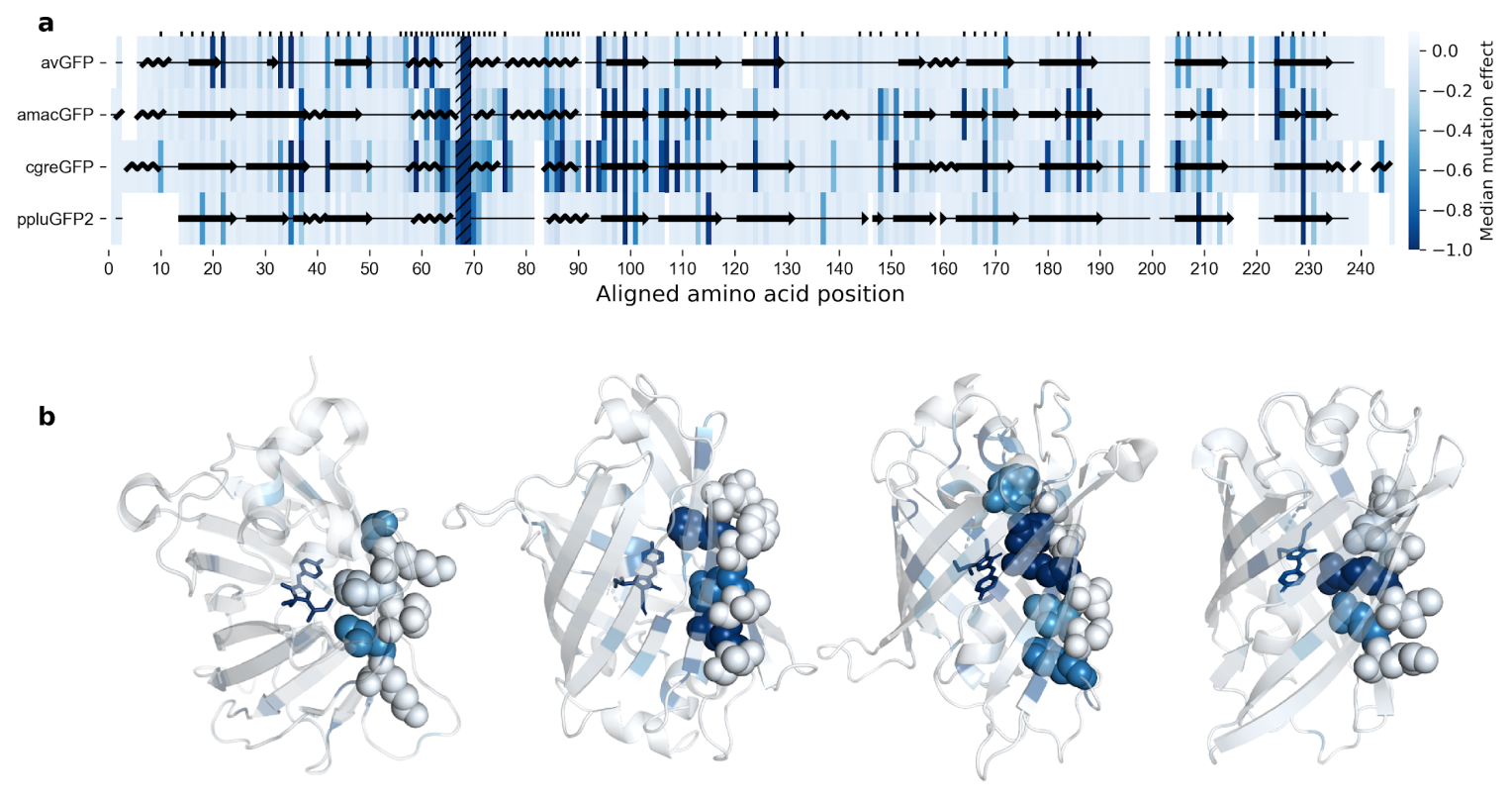

Figure S4. Effects of mutations across the GFP sequences. a, Median effects of single mutations according to sequence position. Amino acid residues on one single strand of the beta barrel of GFP monomers. The chromophore sites are hatched (positions 67-69) and sites with buried amino acid side chains are labeled with tick marks. The overlaid protein secondary structure was obtained with Pymol from the respective crystal structures. $\mathbf{b}$, Median effects of single mutations visualized on protein 3D structures, left-to-right: avGFP (2WUR), amacGFP (7LG4), cgreGFP (2HPW), and ppluGFP2 (2G3O). In each case, a single example beta sheet is represented as spheres in order to better illustrate the difference in mutational effects on residues with internally- versus externally-oriented side chains.

We compared the fluorescence of each genotype to the expected level under an assumption that each mutation influences fluorescence level independently, i.e. without any epistasis (eq. 1):

$$
\text { epistasis }=\text { Effect }_{\text {observed }}-\text { Effect }_{\text {expected }}=\left(F_{m}-F_{w t}\right)-\sum_{i}\left(F_{i}-F_{w t}\right) \cdot x_{i}
$$

Where $\mathrm{F}_{\mathrm{i}}, \mathrm{F}_{m} \mathrm{~F}_{\mathrm{wt}}$ are measured levels of fluorescence of a genotype with a single mutation $i$, of genotype $m$, or of the wildtype sequence, respectively, and $x_{i}=1$ when mutation $i$ is contained within the genotype $m$ and $x_{i}=0$ when it is not. We then calculated the fraction of genotypes that do not require epistatic interactions to predict their fluorescence. On all four fitness peaks, genotypes with two mutations away from the wildtype sequence rarely exhibited any epistatic interactions. However, a striking difference between the fitness peaks was observed when considering genotypes with multiple mutations. The level of fluorescence for a vast majority of genotypes with $>5$ mutations cannot be explained without epistasis in sharp fitness peaks, avGFP and cgreGFP. By contrast, few genotypes 
with $>5$ mutations in flat fitness peaks required epistasis to explain their fluorescence level, with amacGFP requiring almost no epistasis at all (Figure 2d, Figure S5). Interestingly, the sharpness of the fitness peaks and the concomitant extent of epistatic interactions did not correlate with the sequence divergence between the fitness peaks. Indeed, the two closest sequences (82\% identity), derived from the same genus, are the sharp, epistatic avGFP peak and the flat, non-epistatic amacGFP peak (Figure 2d).
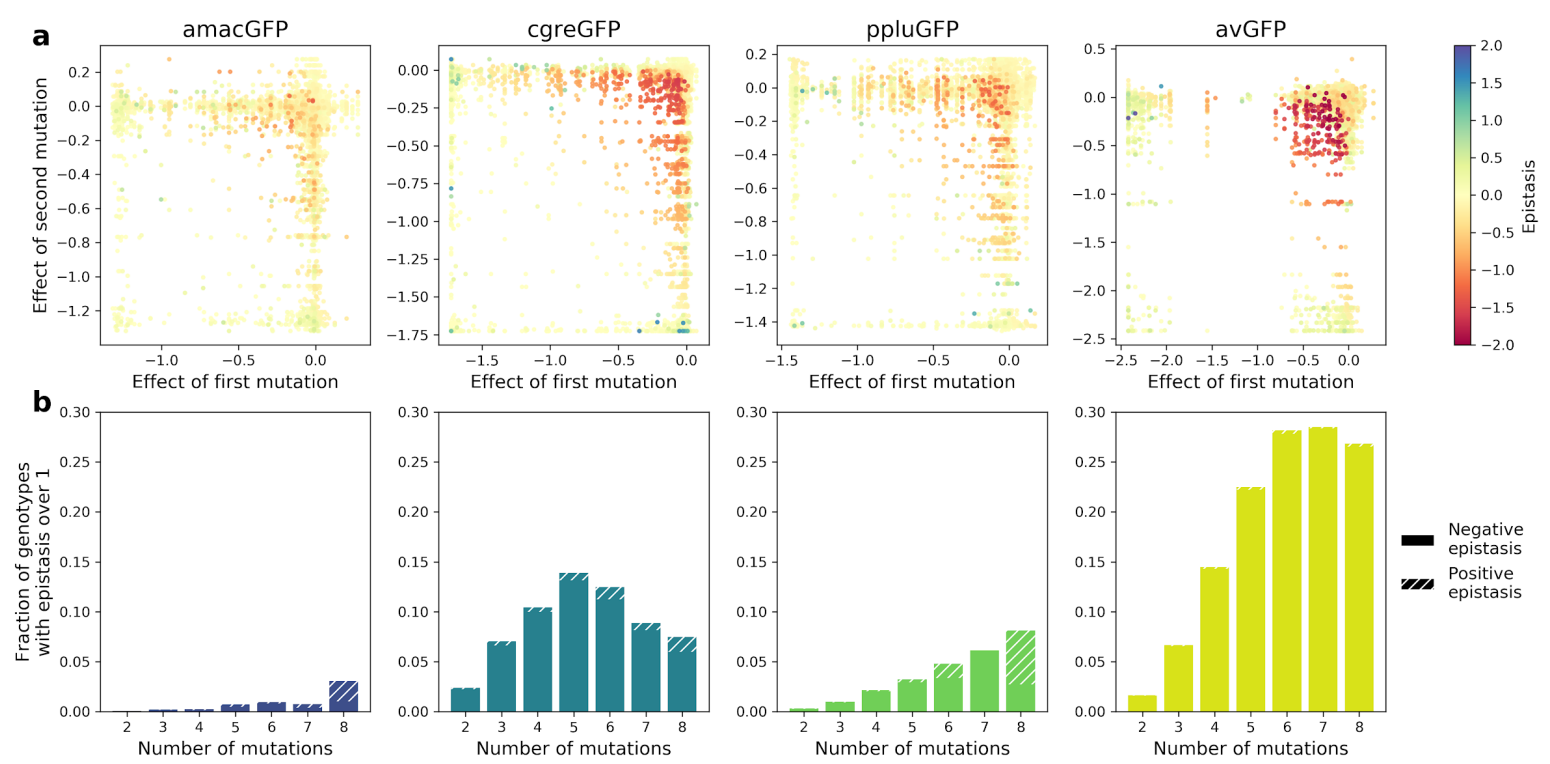

Figure S5. Epistatic interactions of mutations in GFP. a, Epistasis in genotypes with two mutations, highlighting negative epistatic interactions between individually neutral or slightly deleterious mutations. b, Fraction of genotypes that display strong epistasis at different distances from the wildtype.

Flat fitness peaks correspond to mutationally robust proteins, those that are capable of withstanding multiple mutations without losing function, while sharp fitness peaks correspond to mutationally fragile ones. The observed differences in mutational robustness of different proteins may be explained by thermodynamic stability (Bershtein et al., 2006; Echave and Wilke, 2017; Gong et al., 2013; Kurahashi et al., 2018; Poelwijk et al., 2019; Sarkisyan et al., 2016). Therefore, we performed an array of assays aimed at the biophysical characterisation of the four wildtype proteins and an additional genotype, amacGFP:V12L, which differed from amacGFP by the V12L mutation that was extremely common in the amacGFP mutant library. We have assayed the thermal stability of the proteins, using Differential Scanning Fluorimetry (DSF), Differential Scanning Calorimetry (DSC), Circular dichroism (CD), as well as simple measurements of fluorescence in a qPCR machine at different temperatures. We also assayed refolding kinetics of urea-denatured proteins(Pédelacq et al., 2006). Finally, we have assessed oligomeric states of each of the proteins using multi-angle light scattering with size-exclusion chromatography (SEC-MALS). 
The different methods yielded complementary results (Figure S6; Table 2). Specifically, we observed that the most mutationally fragile protein, cgreGFP is also the most kinetically unstable protein and the most mutationally robust protein, ppluGFP2, was also the most kinetically stable (Table 2; Figure S6; Figure S7d-e). These data tentatively suggest that the shape of the GFP fitness peaks, as characterized by mutational robustness, may be shaped by the underlying protein stability. This relationship does not appear to be perfect, as the mutationally fragile avGFP is stable, while amacGFP has mutational robustness comparable to ppluGFP2 (Table 1), but a substantially lower stability (Table 2). Indeed, there may be other factors that influence this relationship, such as the oligomeric protein state and the propensity of the mutant genotypes to aggregate. Indeed, avGFP is the only exclusive monomer from among the four wildtype sequences (Table 2; Figure S8), ppluGFP2 is exclusively tetrameric. The propensity for aggregation also appears variable between the genotypes, with amacGFP showing the highest aggregation of non-fluorescent genotypes (Figure S8).
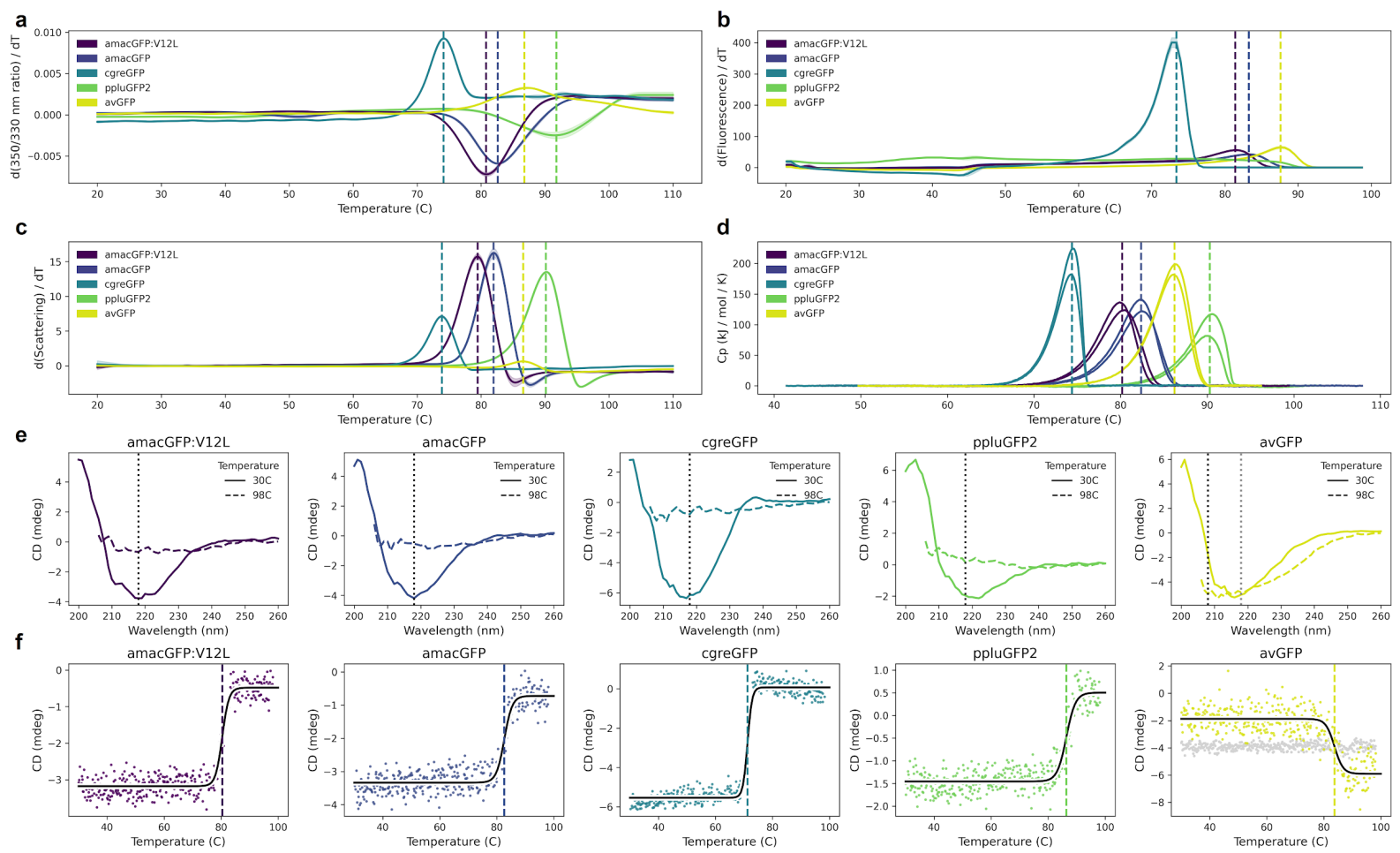

Figure S6. Thermal sensitivity of GFP orthologs. a, Thermal unfolding measured by differential scanning fluorimetry (DSF) showing the first derivative of the ratio of 350/330 $\mathrm{nm}$ emission. Shaded areas indicate standard deviation of triplicates. $\mathbf{b}$, Melting curves of green fluorescence emission $(510 \mathrm{~nm})$ as a function of temperature measured on a qPCR machine. Shaded areas indicate standard deviations of eight technical replicates. c, Thermal aggregation measured by DSF showing the first derivative of the light scattering. 
Shaded areas indicate standard deviation of triplicates. d, Specific heat capacities measured by differential scanning calorimetry in duplicate. e, Circular dichroism (CD) spectra measured before $\left(30^{\circ} \mathrm{C}\right)$ and after $\left(98^{\circ} \mathrm{C}\right)$ with the melting curves depicted in (f) where vertical dotted lines indicate the monitored wavelength in (f). f, CD melting curves monitored at $218 \mathrm{~nm}$ (and additionally $208 \mathrm{~nm}$ in the case of avGFP, where $218 \mathrm{~nm}$ did not show a transition), fitted with a logistic curve. In (a), (b), (c), (d), (f), vertical dashed lines indicate the melting temperature, except ppluGFP2 in (b). In (a), (b), (d), (f), temperature was increased at a rate of $1^{\circ} \mathrm{C}$ per minute, in (c), at a rate of $\sim 2^{\circ} \mathrm{C}$ per minute, the slowest allowed by the LightCycler. 
Table 2. Biophysical and biochemical characterisation of wildtype GFPs.

\begin{tabular}{|c|c|c|c|c|c|}
\hline & amacGFP:V14L & amacGFP & cgreGFP & ppluGFP & avGFP \\
\hline Unfolding Tm (DSF) & $80.8^{\circ} \mathrm{C}$ & $82.6^{\circ} \mathrm{C}$ & $74.1^{\circ} \mathrm{C}$ & $91.8^{\circ} \mathrm{C}$ & $86.8^{\circ} \mathrm{C}$ \\
\hline Aggregation Tm (DSF) & $79.5^{\circ} \mathrm{C}$ & $82.0^{\circ} \mathrm{C}$ & $73.9^{\circ} \mathrm{C}$ & $90.2^{\circ} \mathrm{C}$ & $86.6^{\circ} \mathrm{C}$ \\
\hline $\operatorname{Tm}(\mathrm{CD})$ & $80.4^{\circ} \mathrm{C}$ & $82.6^{\circ} \mathrm{C}$ & $71.2^{\circ} \mathrm{C}$ & $86.4^{\circ} \mathrm{C}$ & $83.7^{\circ} \mathrm{C}$ \\
\hline Transition slope (CD) & 0.86 & 0.72 & 1.27 & 0.63 & 0.67 \\
\hline $\operatorname{Tm}(\mathrm{DSC})$ & $80.2^{\circ} \mathrm{C}$ & $82.4^{\circ} \mathrm{C}$ & $72.9^{\circ} \mathrm{C}$ & $90.3^{\circ} \mathrm{C}$ & $86.3^{\circ} \mathrm{C}$ \\
\hline Enthalpy of denaturation (DSC) & $744 \mathrm{~kJ} / \mathrm{mol}$ & $768 \mathrm{~kJ} / \mathrm{mol}$ & $755 \mathrm{~kJ} / \mathrm{mol}$ & $515 \mathrm{~kJ} / \mathrm{mol}$ & $1012 \mathrm{~kJ} / \mathrm{mol}$ \\
\hline Fluorescence loss Tm (qPCR) & $81.1^{\circ} \mathrm{C}$ & $82.6^{\circ} \mathrm{C}$ & $72.9^{\circ} \mathrm{C}$ & - & $87.5^{\circ} \mathrm{C}$ \\
\hline Urea denaturation: initial rate* & -0.87 & -0.35 & -0.18 & -0.02 & -0.009 \\
\hline $\begin{array}{l}\text { Kinetic parameters for urea } \\
\text { denaturation curves* }\end{array}$ & $\begin{array}{l}a_{1}=0.71 \\
k_{1}=0.96 \mathrm{~h}^{-1} \\
a_{2}=0.28 \\
k_{2}=0.25 \mathrm{~h}^{-1}\end{array}$ & $\begin{array}{l}a_{1}=0.52 \\
k_{1}=0.54 \mathrm{~h}^{-1} \\
a_{2}=0.43 \\
k_{2}=0.12 \mathrm{~h}^{-1}\end{array}$ & - & $\begin{array}{l}a_{1}=0.92 \\
k_{1}=0.02 \mathrm{~h}^{-1}\end{array}$ & $\begin{array}{l}a_{1}=0.92 \\
k_{1}=0.01 \mathrm{~h}^{-1}\end{array}$ \\
\hline Refolding: initial rate ${ }^{\star *}$ & 0.01 & 0.01 & 0.000014 & 0.05 & 0.007 \\
\hline $\begin{array}{l}\text { Kinetic parameters for refolding } \\
\text { curves* }\end{array}$ & $\begin{array}{l}a_{1}=-0.35 \\
k_{1}=0.025 \mathrm{~s}^{-1} \\
a_{2}=-0.36 \\
k_{2}=0.005 \mathrm{~s}^{-1} \\
a_{3}=-0.38 \\
k_{3}=0.001 \mathrm{~s}^{-1}\end{array}$ & $\begin{array}{l}a_{1}=-0.057 \\
k_{1}=0.057 \mathrm{~s}^{-1} \\
a_{2}=-0.39 \\
k_{2}=0.013 \mathrm{~s}^{-1} \\
a_{3}=-0.63 \\
k_{3}=0.002 \mathrm{~s}^{-1}\end{array}$ & $\begin{array}{l}a_{1}=0.16 \\
k_{1}=0.036 \mathrm{~s}^{-1} \\
a_{2}=-0.45 \\
k_{2}=0.01 \mathrm{~s}^{-1} \\
a_{3}=-0.87 \\
k_{3}=0.001 \mathrm{~s}^{-1}\end{array}$ & $\begin{array}{l}a_{1}=-0.32 \\
k_{1}=0.14 \mathrm{~s}^{-1} \\
a_{2}=-0.45 \\
k_{2}=0.02 \mathrm{~s}^{-1} \\
a_{3}=-0.21 \\
k_{3}=0.003 \mathrm{~s}^{-1}\end{array}$ & $\begin{array}{l}a_{1}=-0.4 \\
k_{1}=0.016 \mathrm{~s}^{-1} \\
a_{2}=-0.36 \\
k_{2}=0.001 \mathrm{~s}^{-1} \\
a_{3}=-0.31 \\
k_{3}=0.001 \mathrm{~s}^{-1}\end{array}$ \\
\hline Expected monomer size & $28.1 \mathrm{kDa}$ & $28.1 \mathrm{kDa}$ & $27.4 \mathrm{kDa}$ & $25.7 \mathrm{kDa}$ & $27.9 \mathrm{kDa}$ \\
\hline Primary oligomeric state (SEC-MALS) & $\begin{array}{l}\text { Monomer (67\%), } \\
\text { dimer (31\%) }\end{array}$ & $\begin{array}{l}\text { Monomer (51\%), } \\
\text { dimer (46\%) }\end{array}$ & Dimer (>99\%) & Tetramer (>97\%) & $\begin{array}{l}\text { Monomer } \\
(>99 \%)\end{array}$ \\
\hline
\end{tabular}

* Curves monitoring loss of fluorescence in 9M urea were fitted with two exponential functions in the case of amacGFP and amacGFP:V12L and one exponential function for avGFP and ppluGFP, while cgreGFP fluorescence loss could not be well modeled using only exponential functions (see Figure S7c). Initial rates were estimated by calculating the derivative at time $\mathrm{t}=0$.

** Curves monitoring the recovery of fluorescence after urea denaturation over the course of 20 minutes were fitted with three exponential functions (see Figure S7d). Initial rates were estimated by calculating the derivative at time $\mathrm{t}=0$. 

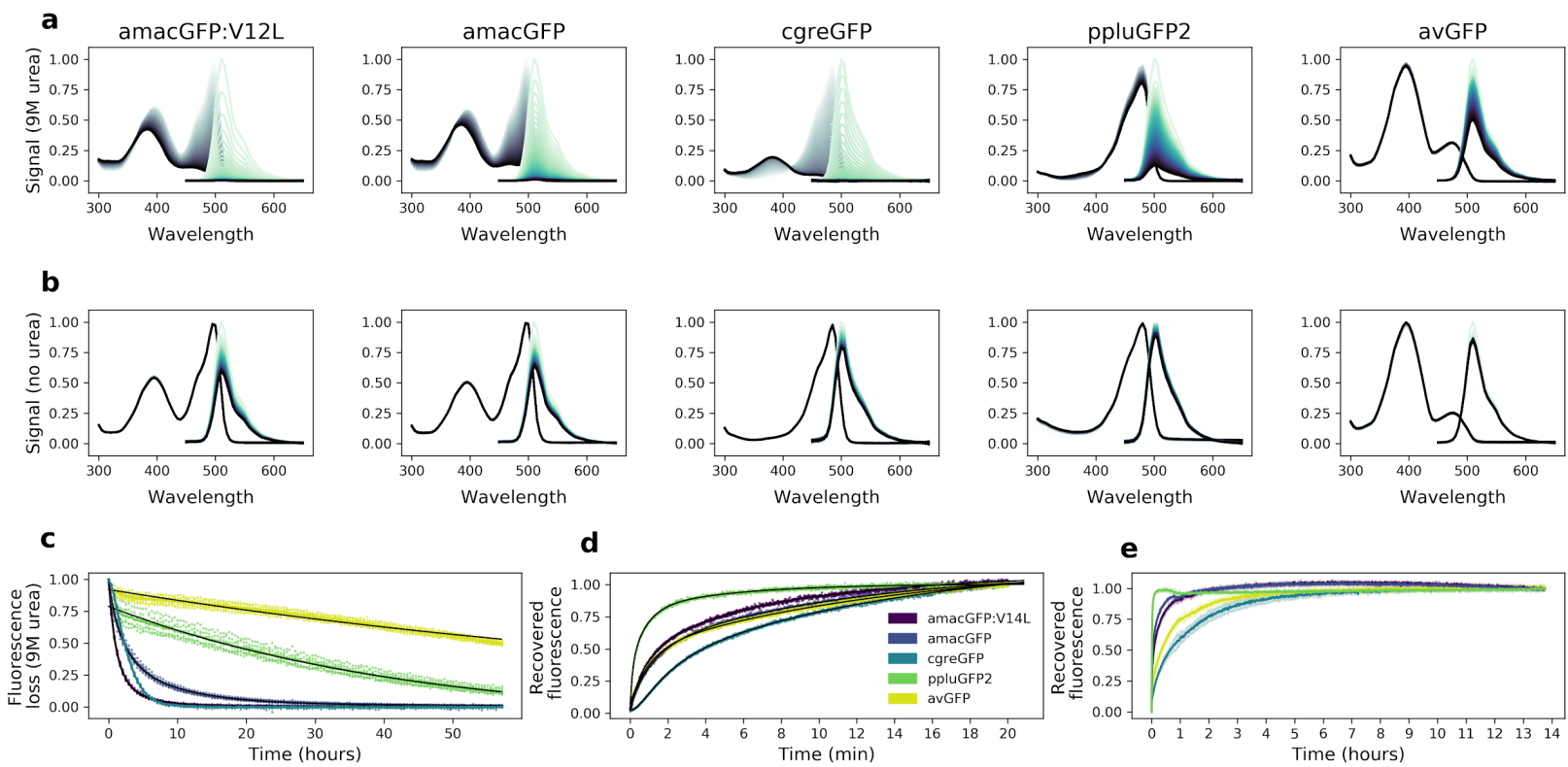

d

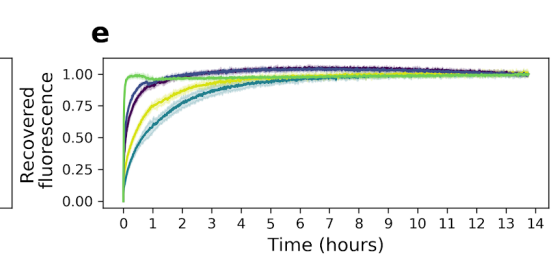

Figure S7. Urea denaturation and refolding of orthologues. a, Absorbance (grey) and fluorescence (green) spectra of purified protein in $9 \mathrm{M}$ urea, or $\mathbf{b}, 1 \mathrm{x}$ PBS, measured at $42^{\circ} \mathrm{C}$ every 30 minutes for 60 hours; darker lines correspond to later time points. Plotted values are the means of eight technical replicates. c, Decrease in fluorescence over time during exposure to 9M urea. avGFP and ppluGFP fluorescence loss curves are fitted with one exponential function $\left(f(x)=a_{0}+a_{1} \cdot e^{-k 1 \cdot x}\right)$, amacGFP and amacGFP:V12L curves are fitted with two exponential functions (black lines), while a good fit using only exponential functions could not be achieved for cgreGFP. Data points from eight technical replicates are shown. d,e, Fluorescence recovery curves of 9M-urea-denatured proteins upon dilution with 20 volumes of 1x PBS. Fluorescence (excitation $485 \mathrm{~nm}$, emission $520 \mathrm{~nm}$ ) was measured every second for twenty minutes in (d), and every 50 seconds for over 13 hours in (e). Values are normalized to the end points. Recovery curves in (d) were fitted with three exponential functions (black). Shaded areas in (e) represent standard deviations of six technical replicates. 

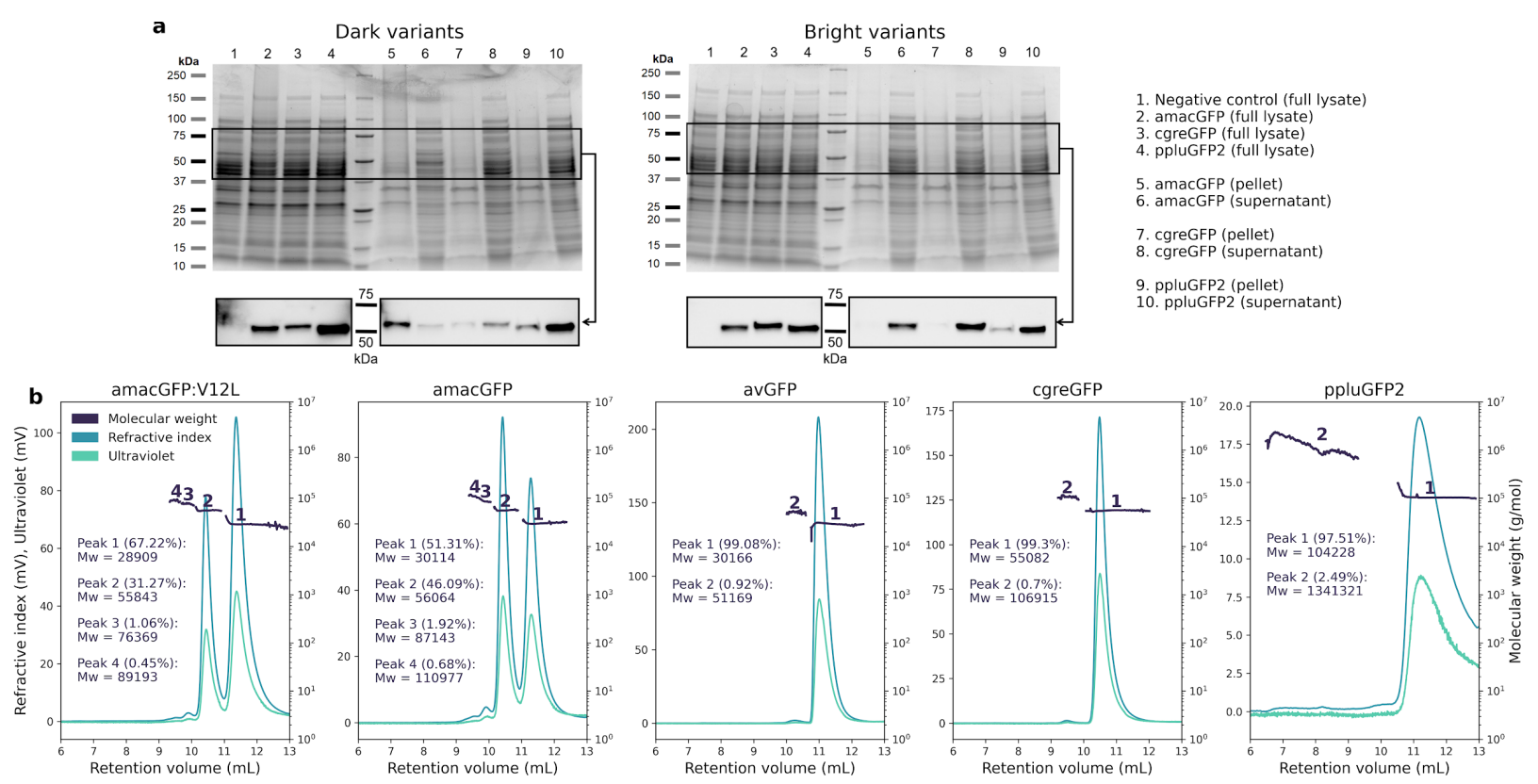

Figure S8. Aggregation and oligomeric states in GFP orthologues. a, Coomassie-stained gels (top) of full lysate, pellet, and supernatant of pooled functional (bright) or non-functional (dark) genome-integrated library variants for amacGFP, cgreGFP, and ppluGFP2. Western blot (bottom) of the same samples using an anti-His-tag antibody, showing different aggregation tendency (i.e. pellet localization) for dark versus bright variants. Expected molecular weight of the mKate2-GFP fusion is 53-56 kDa depending on the GFP ortholog. Negative controls are cells of the same strain, but without integrated GFP constructs. Lane identity in both gels is the same, and displayed on the right. b, SEC-MALS analysis of wild-type proteins, showing protein peaks. Peak analysis is consistent with the following oligomeric states for each gene: amacGFP: primarily monoand dimeric, with small fractions of tri- and/or tetramers; avGFP: primarily monomeric, with a small dimeric fraction; cgreGFP: primarily dimeric, with a small tetrameric fraction; ppluGFP2: primarily tetrameric, with a small fraction forming large aggregates or oligomers. Mw/Mn ratios for all peaks were between 1 and 1.002, with the exception of the large ppluGFP2 aggregates $(\mathrm{Mw} / \mathrm{Mn}=1.147)$.

We then used a computational approach to further explore the relationship between protein stability and the shape of the fitness landscapes. We solved the crystal structure of amacGFP and analysed it along with structures already available for other proteins. We found that mutations causing a substantial reduction of fluorescence tended to have a higher effect on protein stability (Figure S9), estimated by predicted $\Delta \Delta \mathrm{G}$ (Two-sided Mann Whitney $U$ test, $\left.\mathrm{p}<10^{-6}\right)$. Furthermore, we found a statistically significant correlation between predicted $\Delta \Delta \mathrm{G}$ and the effect of a mutation, which was stronger in sharp fitness peaks, avGFP and cgreGFP, and weaker in the flat fitness peaks, amacGFP and ppluGFP2 (Figure S9; Spearman's correlation $\mathrm{r}=0.6$ and $\mathrm{r}=0.3$, respectively). Interestingly, the V12L mutation in amacGFP:V12L appears to have shifted the distribution of the mutation effects, 
substantially increasing the effect of mutations on the barrel lid in proximity to residue 12 , without impacting the overall mutational robustness (Figure S10). Across the whole landscape, epistatically interacting amino acid residues were slightly more likely to be spatially proximal (Melamed et al., 2013; Sarkisyan et al., 2016) and the effect was more pronounced in the flatter fitness peaks (Figure S11). Taken together, these data suggest that the heterogeneity in the shape of the orthologous GFP fitness peaks may be related to the stability of the underlying protein sequences.
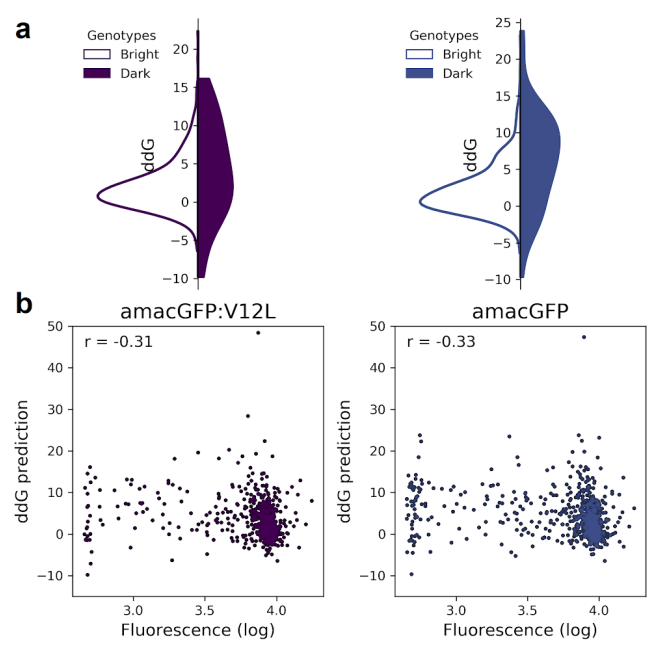
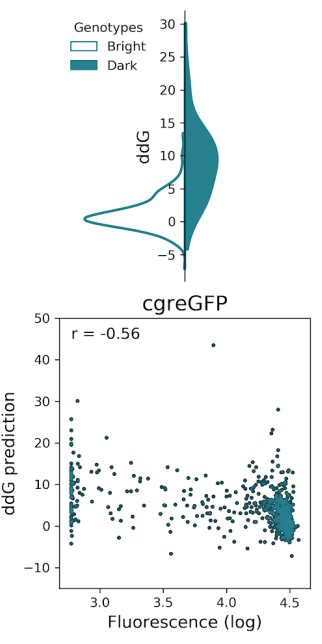
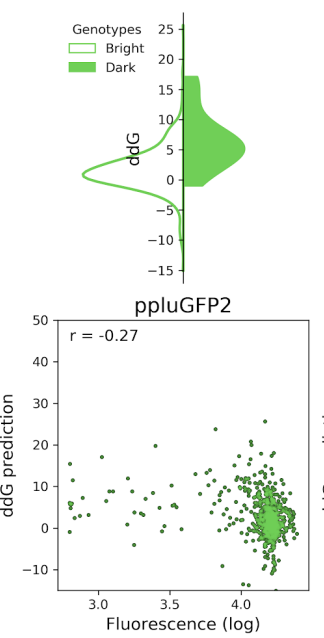
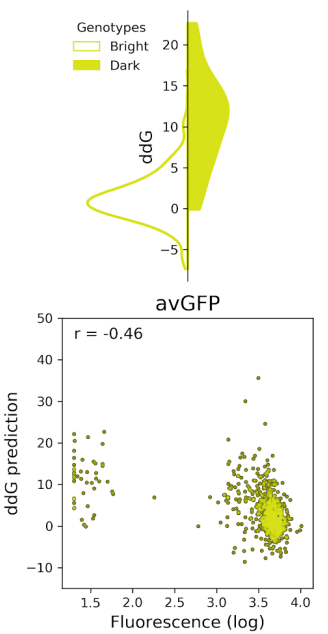

Figure S9. Correlation between fluorescence and ddG predicted by Rosetta. a, Distribution of ddG predictions for single mutations observed to either maintain wildtype-level fluorescence (white) or render a genotype non-functional (colour). Differences between the two distributions were found to be significant for all genes (Two-tailed Mann Whitney U test, $\mathrm{p}=0.02$ for amacGFP:V14L, and $\mathrm{p}<0.00002$ for all other genes). ddG predictions for mutations to and from proline and glycine were not considered. b, Correlation between ddG predictions and observed fluorescence of single mutations. The indicated Spearman's rank correlation coefficient $(r)$ was significant for all genes $(\mathrm{p}<7$. $\left.10^{-15}\right)$. 

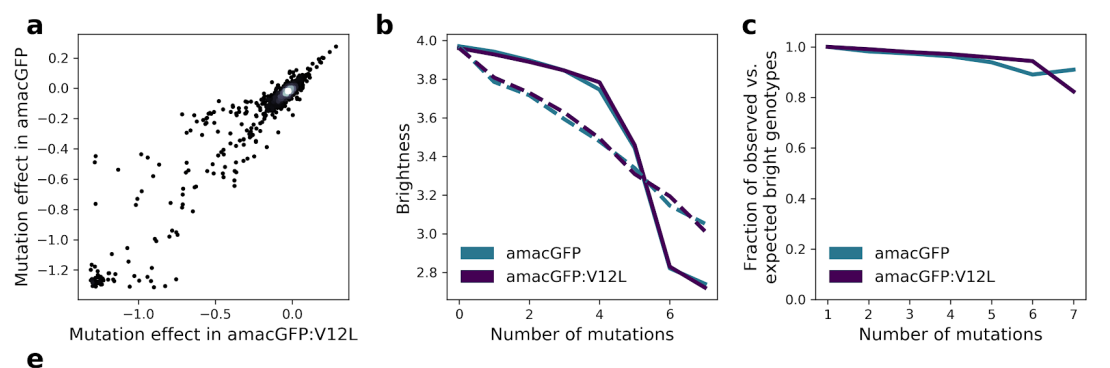

d
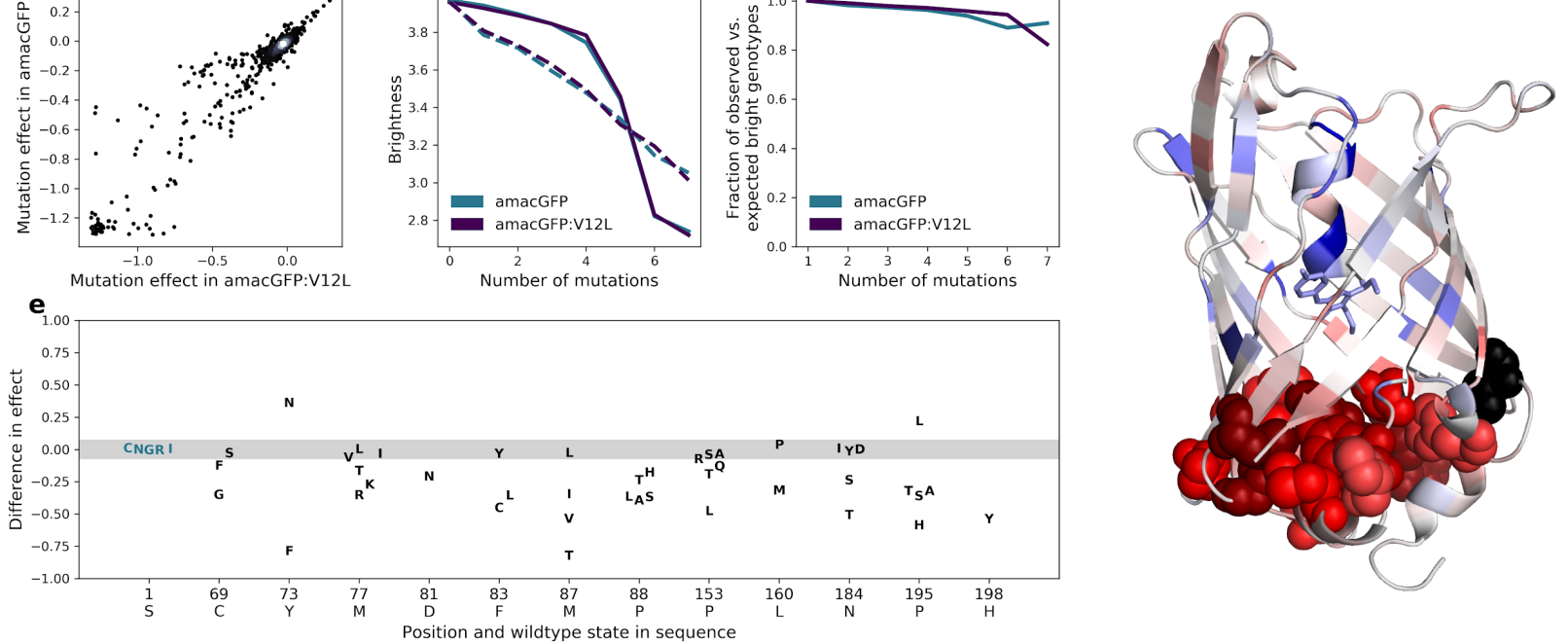

Figure S10. Effects of mutations in amacGFP and amacGFP:V12L. a, Correlation between effects of specific mutations in amacGFP backgrounds with and without the V12L (position 14 in our alignment) mutation (Pearson's $r=0.96$ ). $\mathbf{b}$, Median (solid lines) and mean (dashed lines) fluorescence values of genotypes as a function of the number of mutations relative to amacGFP or amacGFP:V12L sequences. c, Fraction of observed fluorescent genotypes versus expected to be fluorescent under the assumption of no epistasis as a function of the number of mutations away from amacGFP and amacGFP:V12L. $\mathbf{d}$, Median difference of the effect of mutations in amacGFP:V12L relative to amacGFP, colored on the crystal structure of amacGFP. Position 12 is colored black. Residues, mutations of which have a stronger effect in amacGFP:V12L are red, those in which the effect of the mutation is stronger in amacGFP are blue. Atoms of residues with a median difference <-0.1 are shown as spheres. (e) Differences in mutation effect between amacGFP:V12L and amacGFP, at the twelve most affected positions. The majority of mutations have a difference in effect between -0.07 and 0.07 (shaded region, for reference see position 1S). 

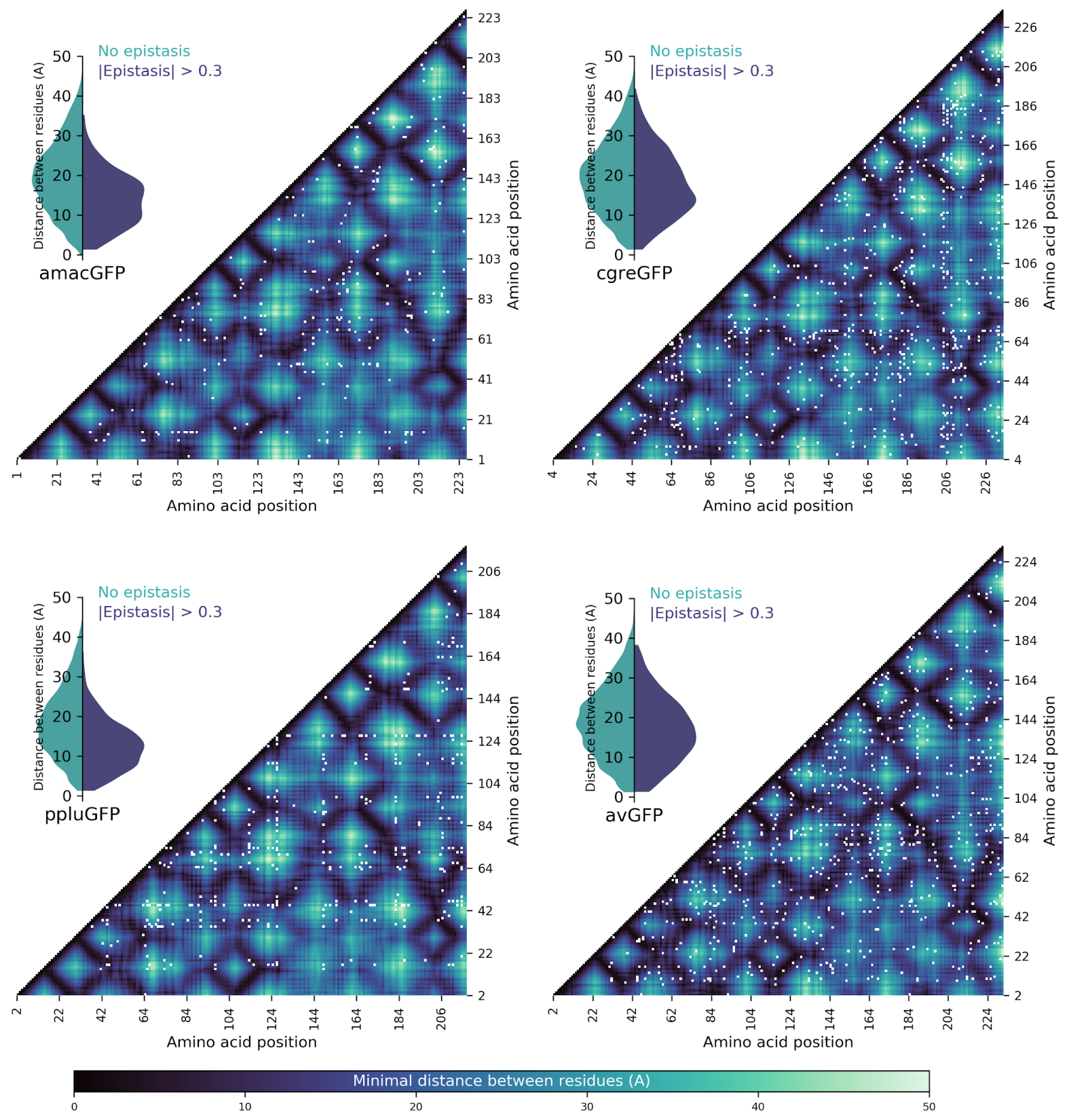

Figure S11. Spatial proximity of amino acid residues and detected pairwise epistasis. Heatmaps show the minimal distance in Angstroms between two residues, with pairs showing epistatic interactions $>0.3$ shown by white dots. Inset violin plots show the distribution of distances between non-epistatic (green) and epistatic (blue) pairs; in all four cases, the epistatic pairs tended to be physically closer (Two-sided Mann-Whitney U-test, $\left.\mathrm{p}<10^{-13}\right)$. 
The apparent lack of a relationship between sequence divergence and fitness peak shape suggests that the shape changes on a scale that is smaller than the distances between the four GFP proteins. Therefore, the difference of the impact of mutations on different fitness peaks should be independent from the sequence divergence between them. We found that the probability that a neutral mutation in one protein becomes deleterious in another one was independent of the sequence divergence (Figure 3a). We then asked if there is a difference in which pairs of sites are interacting epistatically. Interestingly, pairs of epistatically interacting sites were different across all four fitness peaks regardless of the sequence similarity of the proteins (Figure $3 \mathbf{b}$ ). Taken together, these data indicate that underlying rules that determine epistatic interactions and fitness peak shape change on a scale smaller than $20 \%$ of sequence divergence.
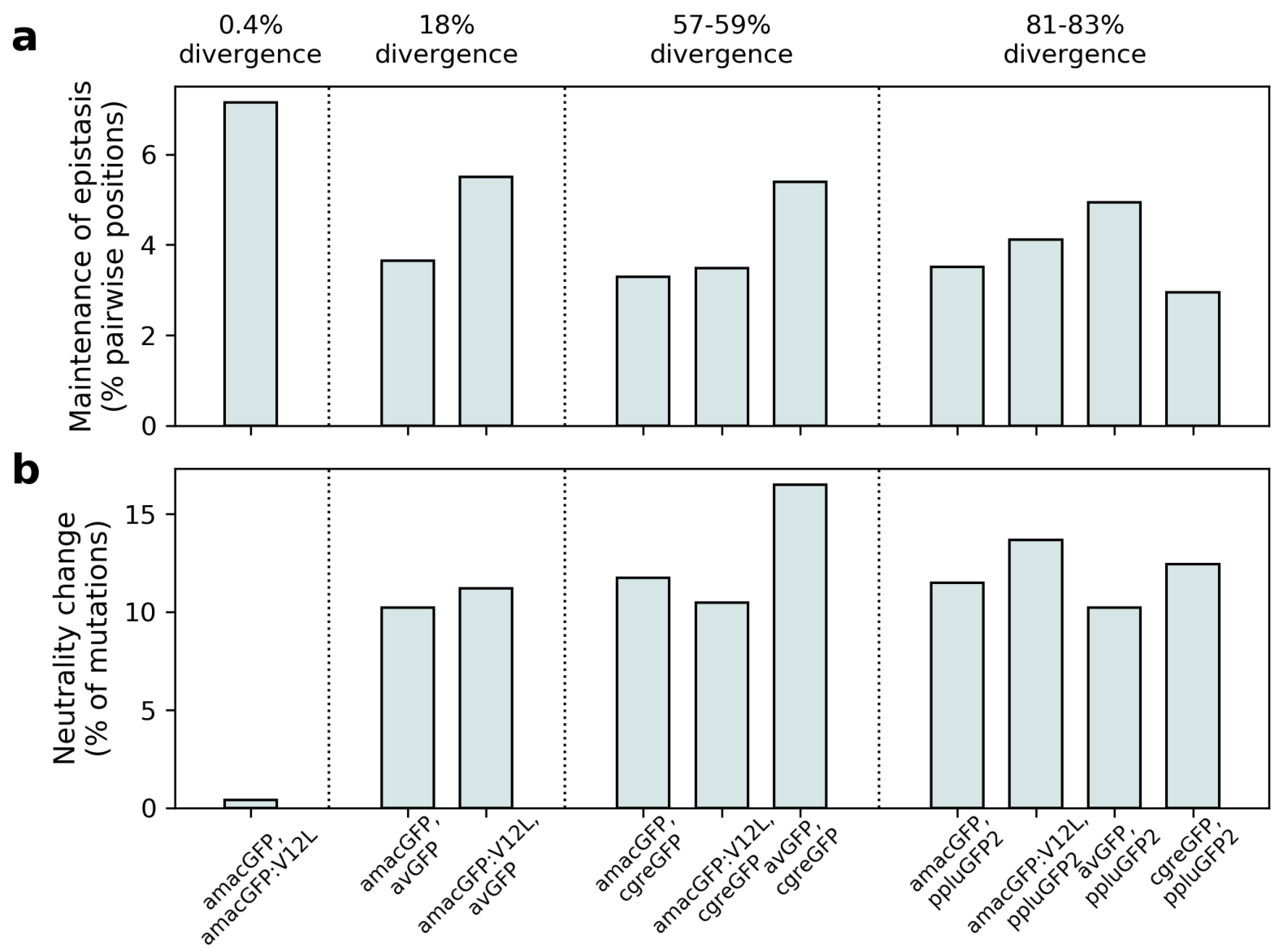

Figure 3. Differences in mutational effects in GFP orthologues. a, The proportion of single amino acid mutations which were observed to be neutral (maintaining fluorescence within two standard deviations of the wildtype level) in one orthologue and deleterious (reducing fluorescence by over five standard deviations) in another, out of all mutations surveyed in both. $\mathbf{b}$, The proportion of pairs of amino acid positions with absolute value of epistasis $>$ 0.3 in both genes, out of all the pairs of positions with epistasis $>0.3$ in at least one of the genes. In (a) and (b), pairs of genes are arranged in order of increasing sequence divergence. 
The identification of two mutationally robust proteins presented an opportunity to predict novel GFP sequences. Two lines of reasoning led us to hypothesize that it would be easier to create functional genotypes by introducing mutations into mutationally robust, rather than fragile, proteins. First, robust proteins had a higher fraction of fit genotypes with $>5$ mutations and, therefore, it should be easier to find other genotypes that are farther away. Second, a robust protein should be more tolerant of mistakes in predictions.

Prediction of functional genotypes many mutations away from known functional sequence is akin to looking for a needle in a haystack. There are $\frac{222 !}{48 ! 174 !} \cdot 19^{48}$ or $\sim 10^{110}$ genotypes that are 48 mutations away from a 222 amino acid long ppluGFP. Out of all of these sequences, only an infinitesimally small proportion is expected to be functional, perhaps as few as $10^{-11}$ [(Keefe and Szostak, 2001)] and finding any appreciable number of these sequences requires extraordinary precision. Therefore, we used a machine learning approach, training neural networks on the genotype-to-phenotype relationships revealed by our data (see Methods, Figure 4a). We split this data into non-overlapping training and validation sets. Models were trained on the training set and after training, model goodness was calculated as the coefficient of determination between predicted and actual fluorescence values for all genotypes in the validation set. We started with a linear model fitted to the one-hot encoded protein sequences. The validation score of the resulting models indicated that between $59 \%$ and $82 \%$ of the variance could be explained in all landscapes by the simple linear contribution of mutations in the protein sequence (Figure S12a). This simple estimate of the fluorescence, which is called fitness potential (Kimura and Crow, 1978; Milkman, 1978), is simply the summed contribution of weighted mutations and does not account for possible interactions between them. We then trained models of increasing capacity and aimed at maximising the validation score while reducing overfitting. In all landscapes, very few intermediate genotypes between the near wildtype and no fluorescence suggests that an abrupt threshold function transforms the fitness potential into the final fluorescence level, as has been observed previously (Pokusaeva et al., 2019; Sarkisyan et al., 2016). Therefore, we decided to train sigmoid models, resulting in the successful capture of an additional $13 \%, 78 \%, 53 \%$ and $39 \%$ of the remaining unexplained variance for amacGFP, avGFP, cgreGFP and ppluGFP2, respectively, compared to the results of the linear model (Figure S12b). This minute transformation of the fitness potential noticeably improves the models' power, especially for the two genes that display the highest levels of epistatic interactions, avGFP and cgreGFP. In order to capture the functions that transform the fitness potential into the predicted fluorescence, we decided to train models with an output subnetwork of several sigmoid nodes (Figure S12c). These functions are shown in Figure S12d. Theorising that models accounting for interactions between residues would push further the predictive power of the models, we optimised the architecture of two-layered networks, one for each dataset using a grid search approach. This resulted in models capturing $0.88,0.95,0.86$ and 0.90 of variance for amacGFP, avGFP, cgreGFP and ppluGFP respectively, as shown in Figure $\mathbf{4 b}$. Using the trained model as the evaluation 
function of a genetic algorithm, we made fitness peak-specific predictions, using the data of each fitness peak to predict fluorescent genotypes containing up to 48 mutations relative to the wildtype sequence.
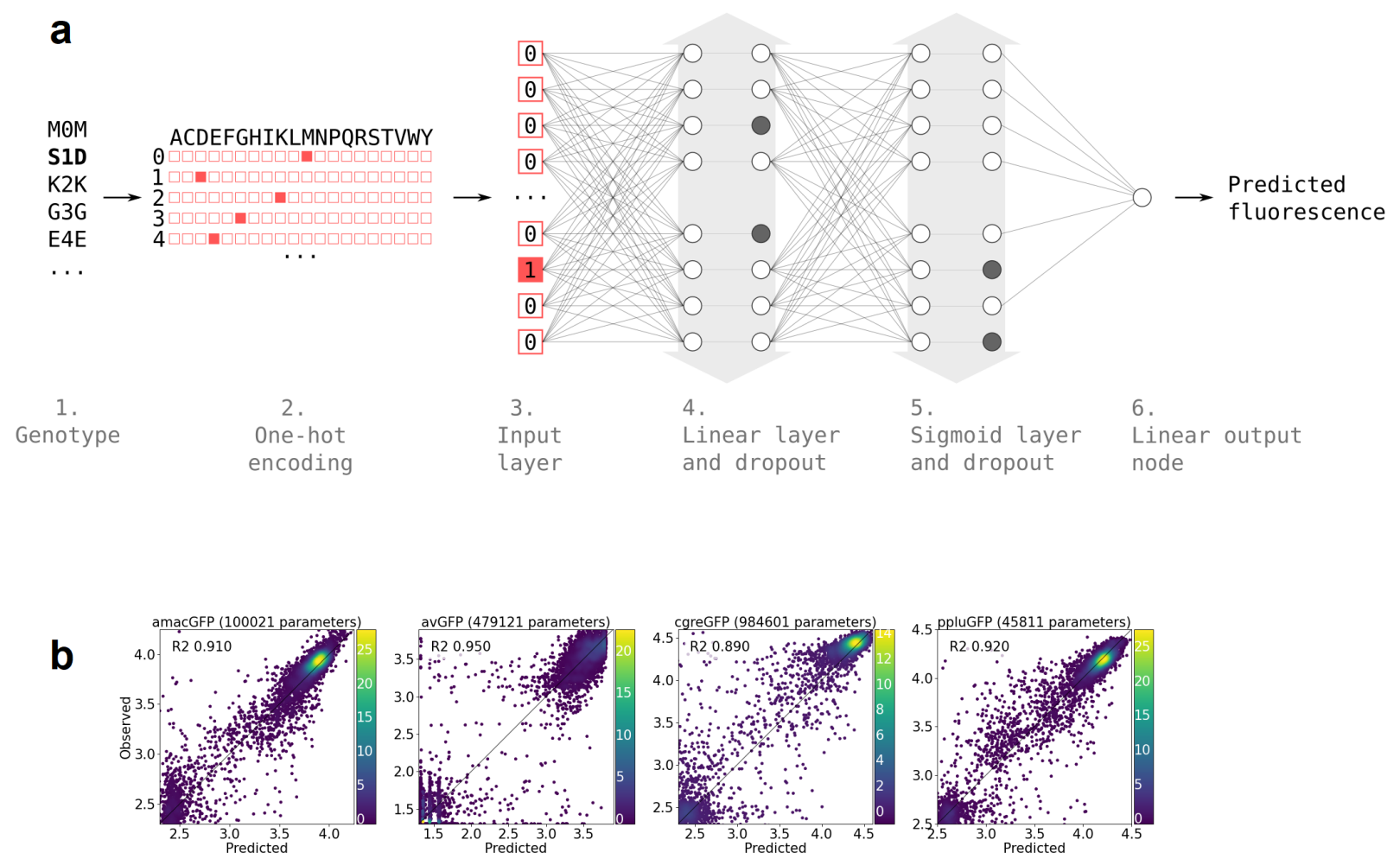

Figure 4. Neural network structure. a, 1. Each genotype in the dataset was denoted by the mutations it contained relative to its parental wildtype sequence. 2. Genotypes were one-hot encoded. For each position in the sequence a binary vector indicated present (red $=1$ ) and absent (white $=0$ ) amino acid states. 3. One-hot encoded sequences were flattened and provided to the neural network as input. 4. The first hidden layer contained linear nodes followed by a dropout layer of the same size. 5 . The second hidden layer contained sigmoid nodes followed by a dropout layer of the same size. Grey arrows indicate layer widths that were optimised by a random search. Greyed-out neurons without output connections represent randomly inactivated neurons in dropout layers. During training, randomly inactivated neurons prevented overfitting. At inference time, randomly inactivated neurons allowed the model to provide different estimates of the fluorescence each time a prediction was run on a genotype. 6. Linear node outputting predicted fluorescence values. For each predicted genotype, the median of several fluorescence estimates was used as the final fluorescence level. $\mathbf{b}$, Correlations between observed and predicted levels of fluorescence with an optimized architecture. Datapoint density is represented in color. 

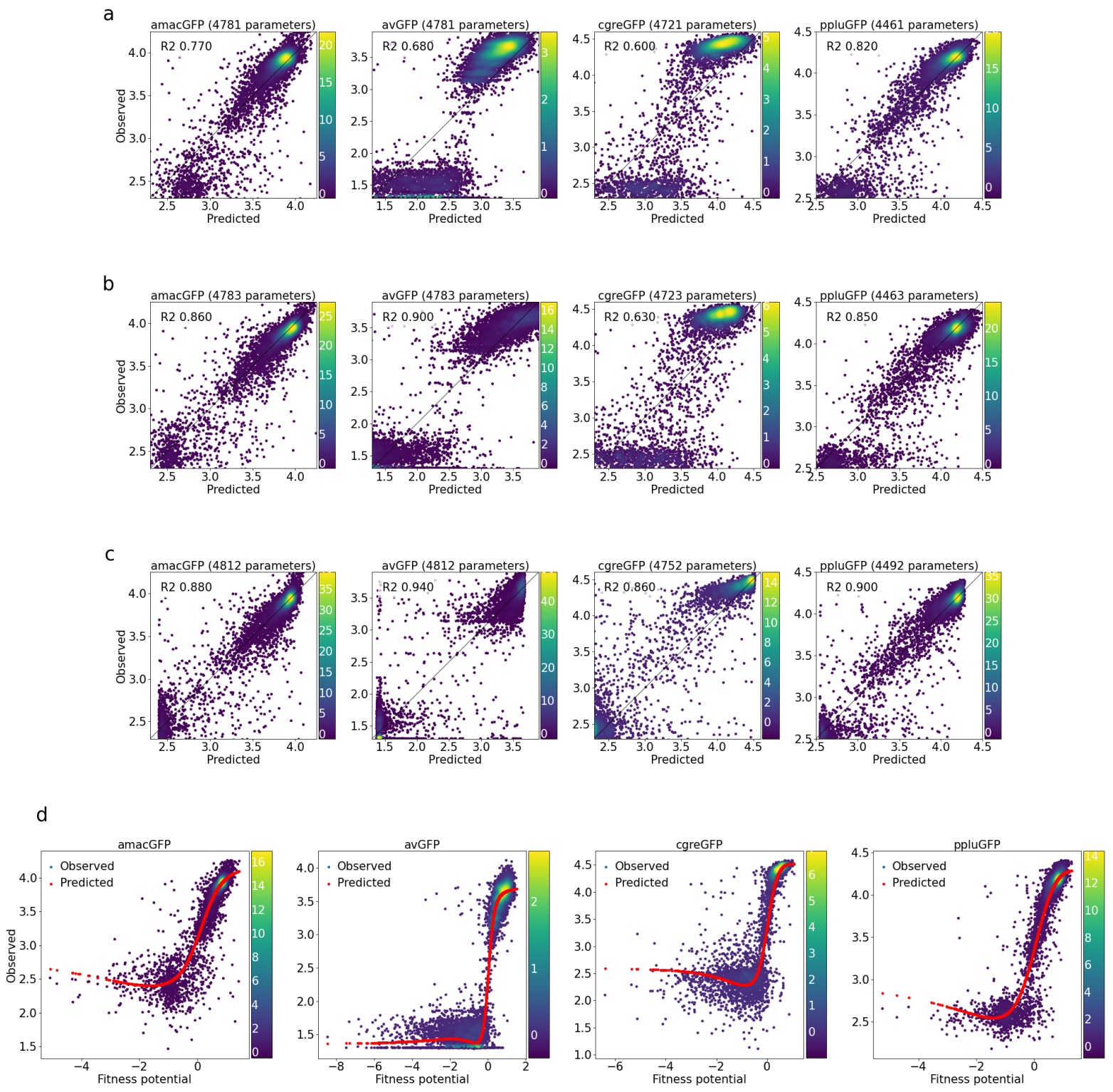

Figure S12. Correlations between observed and predicted levels of fluorescence. a, With a linear model, b, with a linear model and a sigmoid output node, c, with an output subnetwork, and d, non-trivial sigmoidal functions transform fitness potentials into predicted fluorescence. Datapoint density is represented in color.

Amino acids observed in homologous sequences, or extant states, are more likely to be neutral when introduced into a sequence of interest (Pokusaeva et al., 2019) (Figure S13). Therefore, one approach to predict a novel functional sequence would be to prefer the introduction of extant amino acid states. However, we wanted to push the envelope of our predictions in exploring uncharted regions of the GFP fitness landscape, avoiding the 
genotype space between known GFP sequences (space between fitness peaks in Figure 1a). Thus, we aimed to predict genotypes as distant as possible from any known GFP sequences, corresponding to an area of GFP genotype space not known to be explored by evolution. Therefore, for experimental verification from among the predictions made by the machine learning algorithm we selected sequences with the maximum amino acid states not present in any natural GFP.
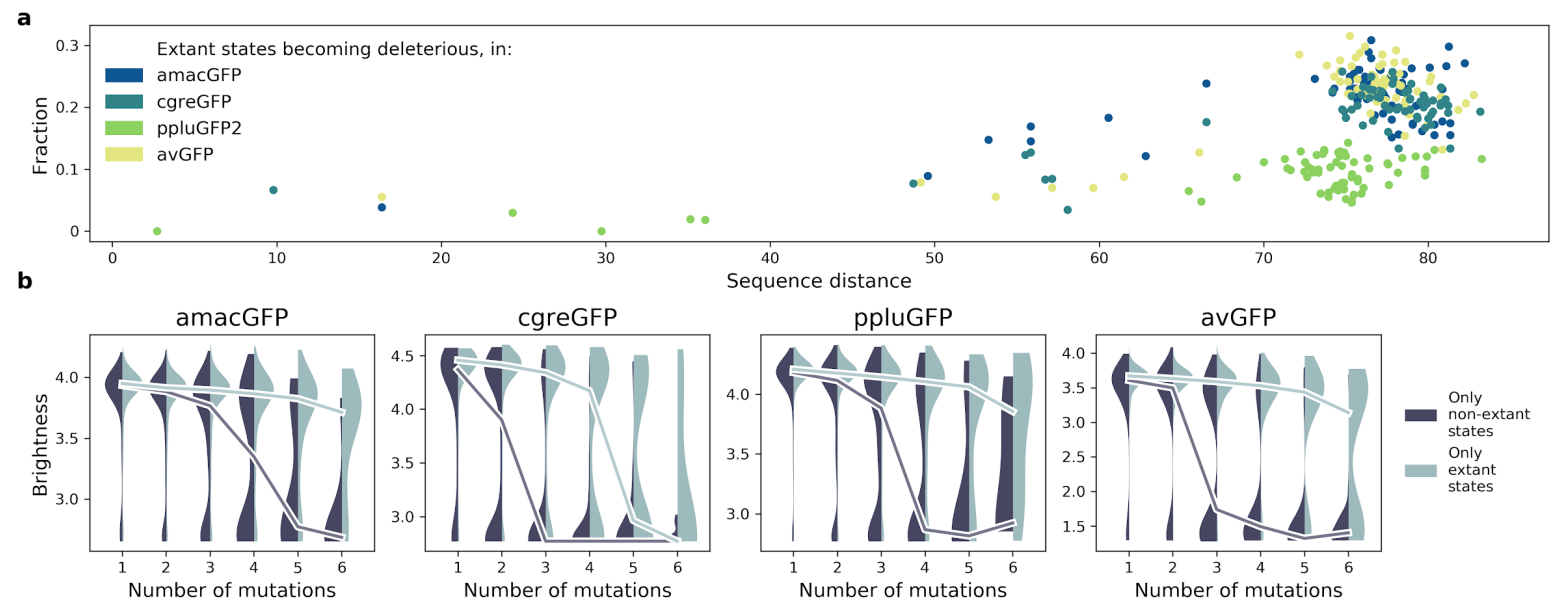

Figure S13. Effect of extant and non-extant mutations. (a) fraction of wildtype states in extant green fluorescent proteins which become deleterious in our data, as a function of the sequence divergence (from 0 to 100) between the two proteins. (b) distribution of fitnesses of mutant genotypes containing only known extant states, or containing no known extant states, at increasing distances from the reference.

Surprisingly, experimental verification showed that the accuracy of our predictions was substantially higher for genotypes predicted by using data from the sharp cgreGFP fitness peak (Figure 5). For genotypes with 48 mutations (>20\% sequence divergence of GFP) our predictions had an $8 \%$ accuracy when using data for the mutationally robust ppluGFP2 and a 50\%-60\% accuracy for the mutationally fragile cgreGFP (Figure 5). These results may be relatively trivial, if the predictions were based on universally neutral mutations(Kondrashov and Kondrashov, 2015), those that are neutral in any GFP sequence. However, three lines of evidence show that our high rate of prediction cannot be explained by universally neutral mutations (also see (Poelwijk et al., 2019)). First, 30\% of these mutations in functional cgreGFP-derived proteins were deleterious in some of the backgrounds we measured (15-20\% in amacGFP and ppluGFP2). Second, the mutations used in successful predictions occur in evolution at a rate two times slower than neutral synonymous substitutions $(0.057$ $\mathrm{dn}$ rate vs $0.11 \mathrm{ds}$ rate, respectively, two-sided Mann-Whitney U-test $\mathrm{p}<0.00001$ ), demonstrating that they are under negative selection. Finally, successful identification of universally neutral mutations would lead to a successful prediction of distant derivatives of any GFP sequence, not just the mutationally fragile cgreGFP. Furthermore, the ML-designed variants derived from the more robust amacGFP and ppluGFP2 proteins were 
rendered non-fluorescent by negative epistasis substantially more frequently than those derived from the fragile and epistatic cgreGFP (Table 1). This suggests that the success of the neural network was dependent on being able to learn epistatic interactions from the data, which were abundant in cgreGFP but rare in amacGFP and ppluGFP2, and to avoid non-favourable epistatic interactions, rather than relying on universally neutral mutations.
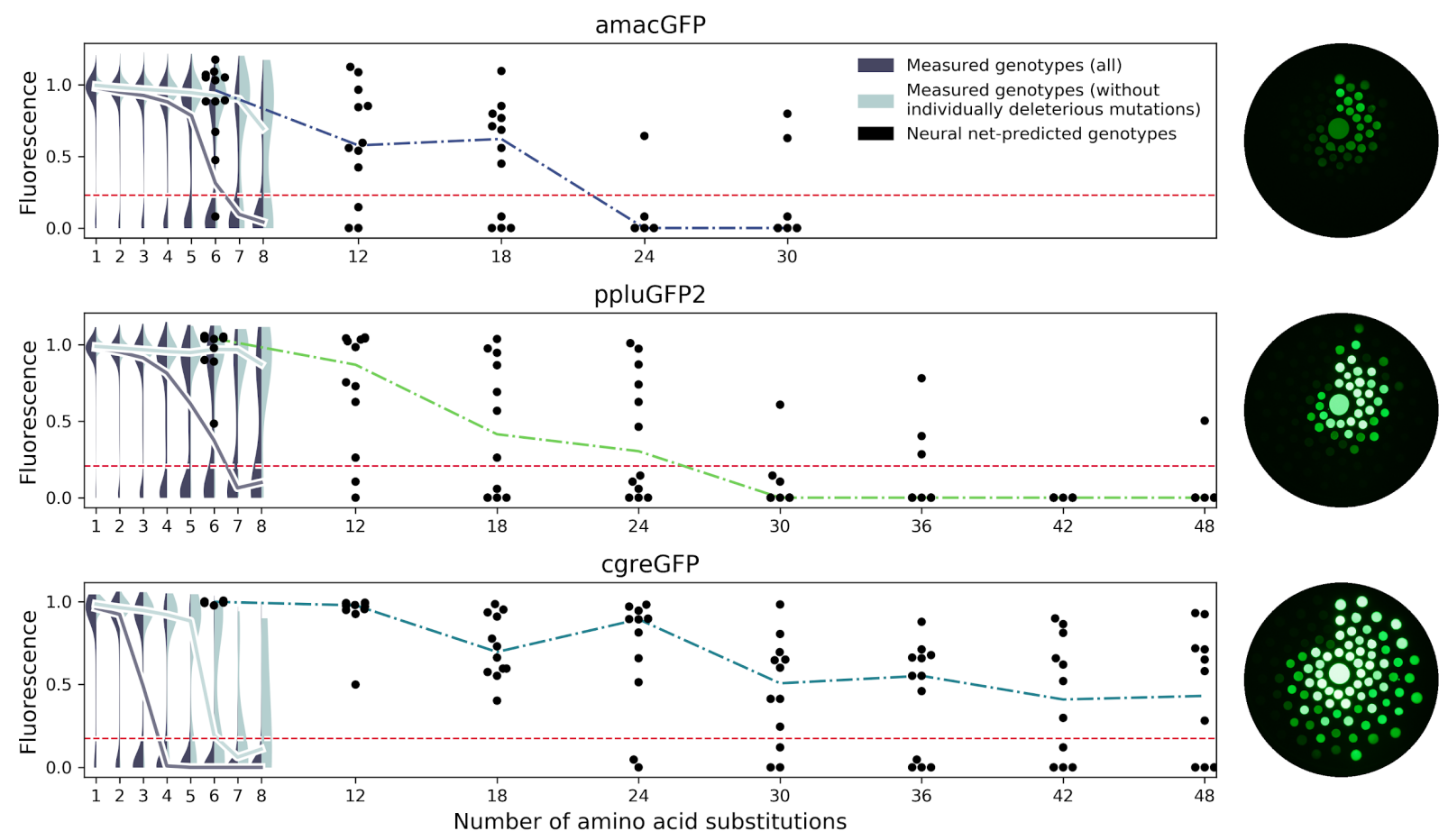

Figure 5. Predicting functional GFP mutants. Violin plots show the distribution of fluorescence of all genotypes (dark grey) and combinations of only individually neutral mutations (light grey). Experimental measurements of the level of fluorescence in genotypes predicted by the neural network are shown in black (12 genotypes per distance). Coloured dashed lines show the median fluorescent values for each group. Red dashed lines indicate the cutoff of detectable fluorescence. Photos of agar plates with E. coli spots expressing predicted GFP variants are shown on the right. Spots of bacteria expressing GFP variants are arranged in circles around the wildtype gene at increasing distance with the number of mutations $(6,12,18,24,30,36,42,48$ mutations). For each group of genotypes, the brightest ones were inoculated at the top, with fluorescence decreasing clockwise.

\section{Discussion}

Experimental survey of the fitness landscape of a protein of interest is increasingly used in protein engineering to discover novel sequences with specific functions (Bryant et al., 2021b; Romero and Arnold, 2009; Russ et al., 2020; Wittmann et al., 2021). While this approach remains challenging for proteins with a function that cannot be easily ascertained in a high-throughput manner (Romero and Arnold, 2009), it is likely to be more widely used in the future due to technological advances of experimental (Romero and Arnold, 2009) and analytical (Wittmann et al., 2021; Wu et al., 2019) tools. Our description of 
heterogeneity of fitness peaks of orthologous GFPs suggests some practical considerations for such surveys of other proteins. Researchers applying such methods to their protein of interest will inevitably have to choose a specific protein sequence to experimentally assay (Romero and Arnold, 2009). When the goal is to discover as many distant functional proteins as possible (i.e. (Bryant et al., 2021b; Romero and Arnold, 2009; Russ et al., 2020; Wittmann et al., 2021)) it may seem natural to select a structurally or mutationally robust protein. Indeed, a robust protein, one that is known to be able to maintain function upon the introduction of many mutations, seems a good starting point to introduce even more mutations. Our results counter this intuition, and our recommendation is to select a fragile protein as the original template for a mutational scan. For the data of the fitness landscape to be useful for a downstream model to predict distant sequences, it has to contain information about epistatic interactions between mutations. Thus, a useful fitness landscape should contain many genotypes that have been rendered non-functional through negative epistatic interactions among a handful of mutations. Our results for cgreGFP demonstrate this principle. Out of 188 genotypes six mutations away from cgreGFP that were expected to be functional by an additive model only 61 (32\%) were actually fluorescent. The rest were non-fluorescent, revealing extensive negative epistasis among those mutations. By contrast, our model correctly predicted 100\% (12/12) of genotypes 6 mutations away from cgreGFP, learning to avoid combinations of individually neutral mutations that combine to create a non-functional genotype. Without this information, such as for amacGFP that shows almost no epistatic interactions within the surveyed genotypes, the model cannot learn which genotypes to avoid (Figure 5). The reported $\sim 20 \%$ prediction accuracy at 40 mutations for sfGFP is also consistent with the sharpness of its fitness peak (Biswas et al., 2018).

Without direct knowledge of mutational robustness of a protein sequence our data indicate that researchers may rely on thermodynamic stability to choose the initial template protein, although the relationship between mutational and thermodynamic robustness may be more complex (Table 2). However, given that for many proteins it is likely to be easier to measure stability than mutational robustness, choosing a structurally unstable protein from several available candidates may prove to be an acceptable compromise.

Despite the success of achieving high accuracy of prediction with our model, there are still substantial limits to the prediction of functional proteins. Indeed, the relatively accurate prediction of functional GFP sequences up to 20\% divergence from cgreGFP does not imply an ability to predict all $\frac{235 !}{20 ! 215 !} \cdot 19^{20}=8.8 \times 10^{53}$ possible functional sequences at this level of divergence. The substantial heterogeneity between fitness peaks of the highly similar avGFP and amacGFP (18\% divergence) suggests that predictions based on a single fitness peak may have lower accuracy of prediction of sequences not governed by the same set of epistatic interactions (Alley et al., 2019; Lee et al., 2018). However, the understanding of the heterogeneity of such predictions would require random sampling of all $8.8 \times 10^{53}$ sequences, which is not presently feasible. 
The heterogeneity of the shape of the fitness peaks and the associated mutational robustness of similar orthologous proteins is remarkable and unexpected. Up to $17 \%$ of all genotypes six random mutations away from the ppluGFP2 wildtype sequence have the same level of fluorescence as the wildtype. By contrast, only $0.9 \%$ of such genotypes derived from the fragile cgreGFP exhibited wildtype fluorescence (Table S1). However, it remains unclear whether this heterogeneity influences protein evolution. It is tempting to suggest that these data indicate that ppluGFP2 is a more "evolvable" protein compared to cgreGFP. However, there are still $2 \times 10^{16}\left(\frac{235 !}{6 ! 229 !} \cdot 19^{6} / 50\right)$ functional genotypes 6 mutations away from cgreGFP, so even such a relatively fragile protein may not be restricted in its long-term evolution (Povolotskaya and Kondrashov, 2010). What fraction of all genotypes $~ 250$ amino acids in length are functional GFPs, and what factors govern differences in the shape of fitness peaks of orthologous proteins, remain unknown.

Data availability. All data and programs relevant to our methodology is available at: https://github.com/aequorea238/Orthologous_GFP_Fitness_Peaks. Cell libraries are available upon reasonable request and subject to a material transfer agreement.

Acknowledgements. We thank Ondřej Draganov, Rodrigo Redondo, Bor Kavčič, Mia Juračić and Andrea Pauli for discussion and technical advice. We thank Anita Testa Salmazo for advice on resin protein purification, Dmitry Bolotin and the Milaboratory (milaboratory.com) for access to computing and storage infrastructure, and Josef Houser and Eva Fujdiarova for technical assistance and data interpretation. Core facility Biomolecular Interactions and Crystallization of CEITEC Masaryk University is gratefully acknowledged for the obtaining of the scientific data presented in this paper. This research was supported by the Scientific Service Units (SSU) of IST-Austria through resources provided by the Bioimaging Facility (BIF), and the Life Science Facility (LSF). MiSeq and HiSeq NGS sequencing was performed by the Next Generation Sequencing Facility at Vienna BioCenter Core Facilities (VBCF), member of the Vienna BioCenter (VBC), Austria. FACS was performed at the BioOptics Facility of the Institute of Molecular Pathology (IMP), Austria. We also thank the Biomolecular Crystallography Facility in the Vanderbilt University Center for Structural Biology. We are grateful to Joel M. Harp for help with X-ray data collection. This work was supported by the ERC Consolidator grant to FAK (771209-CharFL). KSS acknowledges support by President's Grant MK-5405.2021.1.4, the Imperial College Research Fellowship and the MRC London Institute of Medical Sciences (UKRI MC-A658-5QEA0). AF is supported by the Marie Skłodowska-Curie Fellowship (H2020-MSCA-IF-2019, Grant Agreement No. 898203, Project acronym "FLINDIP"). Experiments were partially carried out using equipment provided by the Institute of Bioorganic Chemistry of the Russian Academy of Sciences Core Facility (CKP IBCH). This work was supported by a Russian Science Foundation grant 19-74-10102. This project has received funding from the European Union's Horizon 2020 research and innovation programme under the Marie Skłodowska-Curie Grant Agreement No. 665385. 
Author contributions. KSS, FAK and LGS conceived, designed and supervised the study. LGS performed the experimental work with input from KSS. NGB and JM designed and NGB performed the structural work and analysis. AF and EVP designed and AF performed the bulk of the machine learning analysis. ASM, LGS, AAI, MEAP, KSS, FAK and NGB participated in the data analysis. FAK and LGS wrote the draft with input from all of the other authors.

\section{Methods}

\section{Selection of genes}

Candidate fluorescent proteins were selected based on several criteria: fluorescence in the green spectrum, ability to mature and fluoresce in E. coli under standard culture conditions, and varying degrees of sequence divergence from each other. We also preferred candidates with an available solved crystal structure. Eight genes from six species (Aldersladia magnificus, Aequorea macrodactyla, Clytia gregaria, Clytia hemisphaerica, Pontellina plumata, and Asymmetron lucayanum) were selected as initial candidates. The A. macrodactyla protein contained three point mutations, as the wildtype was previously reported to mature poorly in E. coli (Luo et al., 2006). After testing their expression in E. coli, the three brightest proteins were chosen for further experiments: amacGFP, cgreGFP, and ppluGFP2, respectively from A. macrodactyla, C. gregaria, and P. plumata. Protein sequences of the chosen genes were aligned using the T-Coffee Expresso structural alignment (Armougom et al., 2006).

\section{Golden Gate cloning}

Single-step digestion/ligation Golden Gate protocols were adapted from (Weber et al., 2011). All MoClo reaction mixtures contained 50ng each of insert DNA and destination vector, 10U of T4 DNA ligase (ThermoFisher), 20U of type IIS restriction enzyme (BsaI, BpiI, or BsmBI; ThermoFisher), in T4 ligase buffer at a final concentration of $1 \mathrm{X}$ and volume of 20ul. Thermocycler conditions were as follows: $10 \mathrm{~min}$ at $37^{\circ} \mathrm{C}, 25$ cycles of $1.5 \mathrm{~min}$ at $16^{\circ} \mathrm{C}$ and $3 \mathrm{~min}$ at $37^{\circ} \mathrm{C}, 5 \mathrm{~min}$ at $50^{\circ} \mathrm{C}$, and $10 \mathrm{~min}$ at $80^{\circ} \mathrm{C}$.

\section{Generation of mutant libraries}

Selected genes were ordered as synthetic dsDNA (Twist Biosciences), codon-optimized for bacteria and compatible with common modular cloning (MoClo) standards(Weber et al., 2011). For positions occupied by the same amino acid in different genes, the same codon was used in all genes. The same constant, 20-nucleotide region was included in each gene after the stop codon, for future primer-annealing purposes. All genes were cloned into non-expression storage vectors via MoClo. We generated mutant libraries of each gene via random mutagenesis with the Mutazyme II kit (Agilent), using 200ng of DNA template and eight cycles of mutagenic PCR, in order to achieve an average of $\sim 4$ mutations per clone. Primers (Sigma) included type IIS restriction sites for later cloning, and $20 \mathrm{~N}$ random nucleotide barcodes to label each molecule with a unique identifier, hereafter referred to as "primary barcode". PCR product was gel-purified and cloned into a storage vector via 
MoClo, and transformed into high-efficiency chemically competent E. coli cells (Lucigen E. cloni 10G); post-heat shock recovery time was limited to 15-20 minutes to avoid cell division during recovery and ensure that each resulting colony was the result of an independent transformation event. Up to 150 thousand colonies were recovered and pooled; DNA was extracted following standard maxiprep plasmid extraction protocol (ThermoFisher, GeneJet maxiprep kit) using 2-4 $\mathrm{g}$ of pooled colonies instead of liquid culture. Mutation rates were confirmed by Sanger sequencing (Microsynth) for random 25 clones per library prior to colony pooling.

The mutagenesis kit for creating mutant libraries for amacGFP, cgreGFP and ppluGFP2 was different than that used to create the avGFP mutant library, which led to a slightly different mutational signature, which has not affected our results (Figure S14).
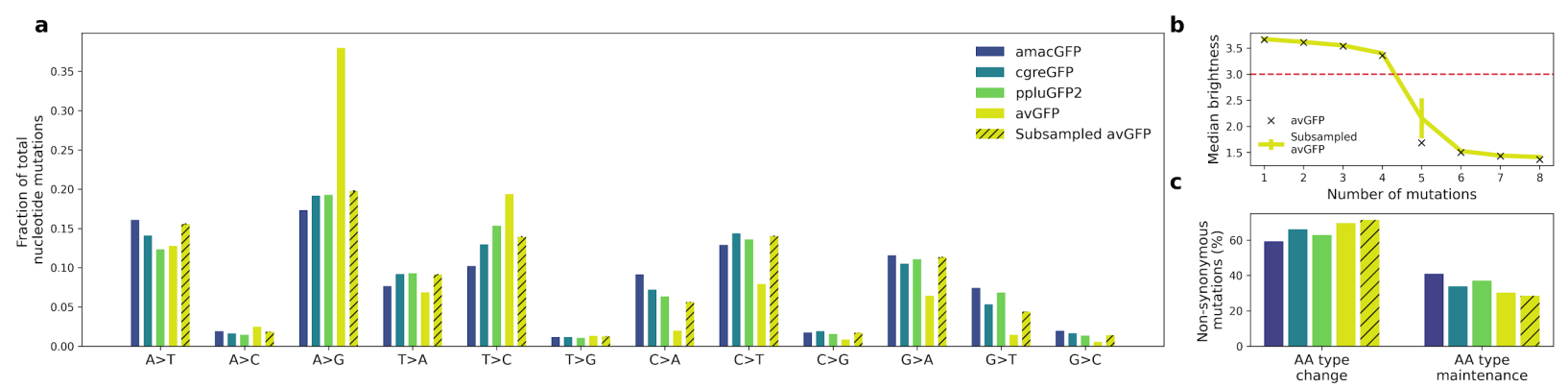

Figure S14. Mutational bias in datasets generated from different mutagenesis strategies.

a, Observed frequencies of nucleotide mutation types in the four landscapes. Libraries of amacGFP, cgreGFP, and ppluGFP2 were generated with the Mutazyme II kit under the same conditions while the avGFP library was generated by (Sarkisyan et al., 2016) employing different dNTP ratios with an in-house error-prone polymerase. "Subsampled avGFP" refers to subsets of 15,000 genotypes of the avGFP library, sampled to mimic the mutational patterns of the Mutazyme-generated libraries. b, Median brightness of genotypes as a function of the number of nucleotide mutations. Black X marks indicate the values for the complete avGFP dataset, and the yellow line shows the values for the subsampled datasets whose mutational patterns are shown in the hatched bars in (a). Error bars indicate standard deviation for 100 simulations. The horizontal red line indicates the threshold used in (Sarkisyan et al., 2016), under which genotypes were considered non-functional. c, Fractions of non-synonymous mutations which result in a significant change in the chemical properties (Creighton and Creighton, 1993) of the amino acid. With the exception of histidine (aromatic and positively charged), we considered amino acids as belonging to one of six categories: positively charged (Arg, Lys, His), negatively charged (Glu, Asp), polar uncharged (Ser, Thr, Gln, Asn), aromatic (Tyr, Trp, Phe, His), aliphatic (Ala, Iso, Leu, Met, Val), and other (Gly, Pro, Cys). A type change was considered to occur when a mutation resulted in the new amino acid belonging to a different category from the original. The difference in percentages between the avGFP ratios and the average 
amacGFP/cgreGFP/ppluGFP ratios were not statistically significant (Fisher's exact test, $\mathrm{p}=$ $0.3)$.

\section{Generation of expression cassettes}

We assembled an expression vector via MoClo from the following parts: a 5' 600bp homology arm to the E. coli genome; an mKate2-LacZ fusion under the T5 promoter, followed by a placeholder sequence flanked by IIS restriction sites, and lambda T0 terminator; a zeocin resistance cassette; and a 3' 600bp homology arm. The placeholder was subsequently replaced by $10 \mathrm{~N}$ random nucleotide barcodes, hereafter referred to as "secondary barcodes", in order to create a library of around 10 thousand expression vector variants differing only by this barcode.

Mutant libraries were then shuttled from their storage vectors into the pooled expression vectors, replacing LacZ with GFP in-frame with mKate2 and allowing for color-based determination of cloning efficiency upon plating with $\mathrm{X}-\mathrm{Gal}$. Final constructs thus expressed GFP mutants as a fusion protein with mKate2, ensuring the two proteins are equimolar inside each cell and allowing mKate2's red fluorescence to be used as a control for GFP expression level (Figure S2). mKate2 was selected as previously (Sarkisyan et al., 2016; Weber et al., 2011) due to its spectral properties (minimum overlap with GFP spectra, and lack of green emission phase during maturation) and monomeric activity, and is separated from GFP by a rigid alpha-helix linker to avoid any potential interactions between the two proteins. An N-terminal 6-His-tag was also included in the mKate2-GFP fusion design.

GFP mutant libraries were shuttled into the expression vector via modular cloning protocols described above. MoClo reactions were transformed into high-efficiency chemically competent E. coli cells (Lucigen E. cloni 10G). Around 800 thousand colonies were recovered; each mutant genotype, identifiable by its primary barcode, is thus expected to be associated with multiple secondary barcodes. This approach created internal replicates for each genotype, with each primary/secondary barcode combination having been the result of independent cloning and transformation events, allowing for independent measurements of the same genotype during a single experimental set-up.

\section{Genome integration}

Genome integration is expected to produce less expression noise compared with expression from a plasmid (Lee et al., 2015). Final expression-ready cassettes were excised from the vector backbone via digestion at SpeI sites flanking the homology arms, and gel-purified. Linear fragments were integrated into a safe harbor in the E. coli chromosome via CRISPR-Cas9-mediated homologous recombination, using a protocol adapted from [(Bassalo et al., 2016)]. In brief, we transformed cells with Court lab's pSIM5, a temperature-inducible plasmid containing genes necessary for homologous recombination, and pX2-Cas9 (Addgene \#85811), an arabinose-inducible Cas9 vector. Cells were grown to 
the exponential phase (OD600 $=0.6$, measured via NanoDrop) at $30^{\circ} \mathrm{C}$ in the presence of $0.2 \%$ arabinose and then heat-shocked at $42^{\circ} \mathrm{C}$ for fifteen minutes to activate pSIM5. We observed increased efficiency when cells were grown with arabinose from the start, rather than only provided with it during the recovery phase. Cells were then placed on ice for 20 minutes, washed thrice with ice-cold distilled water, and electroporated with the linear library DNA as well as the SS9_RNA (Addgene \#71656) vector containing the guide RNA for Cas 9 to target the safe harbor. Cells were plated on $50 \mathrm{mg} / \mathrm{L}$ zeocin plates after two hours of recovery at $30^{\circ} \mathrm{C}$ in $0.2 \%$ arabinose-supplemented $\mathrm{LB}$, grown overnight at $30^{\circ} \mathrm{C}$ and an additional day at room temperature, then recovered from plates and resuspended in LB for sorting. Approximately five million colonies were recovered in each case.

\section{Fluorescence-activated cell sorting}

Resuspended cells were sorted in parallel on two independent BD FACS Aria III cell sorters ("machine A" and "machine B") at a rate of around 20 thousand events per second. Each library was processed independently, but a small amount of wild-type avGFP, amacGFP, cgreGFP, and ppluGFP2 genotypes with known barcodes were added to each library as positive controls and for the purposes of cross-library comparisons. A narrow gate in the red channel was selected, corresponding to a fixed mKate2 expression level, and this was subdivided into eight sub-gates based on green intensity (Figure S2) and these were sorted into eight separate tubes. For each library, around 28 million cells were sorted in total, leading to an estimated average of $\sim 35$ recovered cells for each colony in the pool.

After library sorting, we separately added 5000 cells each of four known barcodes to each tube, to serve as controls for the number of cells sorted and determine how many reads are generated per cell. "Count control" cells were generated separately from the mutant libraries, so their barcodes are not expected to be present in any of the libraries.

The use of mKate 2 controls for the influence of variability in gene expression, but also can be used to control for the impact of mutations on mRNA structure, stability, or translation. Such mutations could be either synonymous or non-synonymous. If mutations had a substantial probability of impacting green fluorescence through mRNA structure, stability or through their impact on translation, we would expect for our data to contain a non-negligible amount of such synonymous mutations. However, since synonymous mutations do not influence fluorescence (Figure S3a), these effects are not present on a detectable level.

\section{Circularization of mutant libraries}

For each gene, approximately $5 \mu \mathrm{g}$ of DNA (mutant libraries in storage vectors) was digested with BsaI and the GFP fragment was recovered via gel purification, leaving known 5' overhangs. A short dsDNA filler sequence with compatible overhangs was used to tie the Nand C-terminal GFP ends together. The filler was obtained by annealing two complementary primers (mixed together in equal amounts, heated to $95^{\circ} \mathrm{C}$, and gradually 
cooled down to $20^{\circ} \mathrm{C}$ at a rate of $0.1^{\circ} \mathrm{C}$ per second using a thermocycler); compatible overhangs with the GFP fragment were generated by BsaI digestion.

Circularization was performed at room temperature in a volume of $500 \mu \mathrm{l}$ 1X T4 DNA ligase buffer, with 100U BsaI and 60U T4 DNA ligase, and starting quantities of $50 \mathrm{ng}$ each of linear GFP library fragment and dsDNA filler. Another $50 \mathrm{ng}$ of each were added every 30 minutes until reaching a combined total amount of $2 \mu \mathrm{g}$. DNA addition was performed gradually in order to minimize the concentration of unligated linear DNA and thereby avoid formation of tandem multimers; once ligated, circular products cannot be cut again due to restriction site destruction. The circular monomer fraction was isolated by gel purification of the appropriate band. Successful circularization was confirmed by PCR.

\section{Preparation of mutant libraries for sequencing of coding region}

Mutant GFP libraries were sequenced via MiSeq 300bp-paired-end Illumina sequencing, performed by the Vienna Biocenter Core Facilities. In order not to exceed the maximum total read length of $600 \mathrm{bp}, \mathrm{N}$-terminal and C-terminal halves - each between 400 and 500 bp - were prepared separately.

C-terminal halves were PCR'd directly from the storage vectors containing the mutant libraries. For N-terminal halves, libraries were first circularized in order to place the barcode adjacent to the start codon. In each case, a first round of 10 PCR cycles was performed, using three pairs of primers incorporating part of the constant region of Illumina TrueSeq adapters; the three pairs differed only by the addition of $1-2 \mathrm{~N}$ bases, in order to create sequence shifts and increase complexity for NGS purposes. These products were gel-purified and used as templates for a further 10 PCR cycles with primers incorporating Illumina indices. Final PCR products were gel-purified, eluted in nuclease-free water and sent for sequencing. Different indices were used for different halves and for different genes, allowing pooling of samples to be sequenced in the same MiSeq lane. A total of 4 lanes were used for MiSeq library sequencing.

\section{MiSeq data processing}

Sample de-multiplexing was performed by the sequencing facility. Raw Illumina sequencing data was converted from .bam to .fastq format using Bamtools; all further processing was performed with custom Python scripts. BioPython was used for pairwise alignments.

MiSeq reads were first checked for the presence of the constant region located in between the stop codon and the barcode; reads lacking this motif were discarded. Barcodes were extracted, corresponding to the 20 nucleotides adjacent to the constant region. Primer sequences were trimmed from all reads. Reads with matching barcodes were pooled, and consensus sequences of the GFP-coding region were obtained independently for the C-terminal and N-terminal halves, as well as for the forward and reverse reads of each half. Barcodes with fewer than 5 reads in one or both halves were discarded, as well as barcodes with less than $80 \%$ agreement for any given position. Consensus sequences of forward and 
reverse reads were merged, then $\mathrm{N}$ - and $\mathrm{C}$-terminal halves were merged to obtain the full coding sequence; barcodes where the expected overlap was less than a $100 \%$ match between sequences to be merged were discarded.

Final coding sequences for each barcode were then compared with the wild-type template by global pairwise alignment and mutations were extracted. Coding nucleotide sequences were translated to obtain amino acid sequences.

\section{Preparation of samples for HiSeq barcode sequencing}

Sorted cells were recovered periodically during sorting, and kept on ice to avoid cell division. In order to increase the genetic material available for PCR, recovered cells were plated on LB-zeocin agar and incubated overnight at $37^{\circ} \mathrm{C}$. Colonies were pooled and mixed and used as PCR template to amplify the barcode region, as we previously found PCRs directly on the sorted cells to be inefficient.

As with MiSeq sample preparation, two rounds of PCR were performed. The first round consisted of 15 cycles (Encyclo polymerase, Evrogen) and used N-shifted primers to increase complexity, and was gel-purified and used as the template for the second round, which consisted of 9 cycles and added full Illumina TrueSeq adapters. Final products were gel-purified and sent for HiSeq SR100 sequencing. Different Illumina indices were used for different samples, allowing pooling of multiple samples into the same sequencing lane. Sample ratios in each lane corresponded approximately to the numbers of sorted cells. A total of four HiSeq lanes were used, with twelve samples per lane.

\section{HiSeq data processing}

As with MiSeq data, HiSeq sample demultiplexing was performed by the sequencing facility, raw data was converted from .bam to .fastq using Bamtools, and further processing was done with custom Python scripts.

HiSeq reads encompassed the barcode region only: a $20 \mathrm{~N}$ primary barcode and a $10 \mathrm{~N}$ secondary barcode, with a 10bp-length constant region in between. Reads lacking the constant motif were discarded. Primary and secondary barcodes were extracted from each read, and reads with matching primary barcodes were pooled. If a secondary barcode had fewer than six reads, and differed from another secondary associated to the same primary by two or fewer nucleotides, it was considered to be the product of sequencing errors and its reads were merged together with the more abundant barcode. For each primary-secondary barcode combination, the distribution of reads across the eight green sorting gates was determined.

In order to estimate the actual number of sorted cells from the number of reads, we used "count control" barcodes mentioned previously: cells of known barcode of which a fixed amount was sorted into each tube. For each gate, the read counts of all barcodes were normalized according to the average "count control" read count of that gate. 


\section{Estimating fluorescence for each genotype from HiSeq sequencing of sorted populations} To determine the genotype distribution across brightness populations we used Illumina HiSeq (single-end 100 bp reads). The fluorescence for each genotype was assessed by fitting the calculated number of cells across the sorting gates to the cumulative density function of normal distribution (provided by scipy.stats.norm Python module), taking into account gate border values for every sorter run (Supplementary Data 1). The fitting was performed with the scipy.optimize.curve_fit Python module, with the initial guess corresponding to the gate with the highest cell count observed. The initial guess for the sigma equaled the width of the gate with maximum cell count. In order to correct for slightly different settings between two FACS machines, the brightness values were matched by linear regression of fluorescence values of known wild-type genotypes.

\section{Genotype data filtering}

Our experimental setup allowed for various sources of replication for each mutant: cells with the same genotype and primary barcode but different secondary barcodes; or the same genotype but different primary barcodes; or the same genotype as well as primary and secondary barcode but sorted on different machines. Such replicates were merged, and assigned a fitness corresponding to the mean of the fitnesses of each individual replicate, weighted by their cell counts (Supplementary Data 2).

Two sources of internal controls allowed us to check data quality: genotypes corresponding to wild-type proteins, known to be bright, and genotypes corresponding to chromophore-mutated variants known to be dark. Due to the physical limitations of FACS, it is not unexpected for some number of cells to be mis-sorted into the wrong gate, but we expect such events to be associated to low read counts, and for mis-sorted cells to be associated to different fitnesses than correctly sorted cells of the same genotype. Therefore, we discarded nucleotide genotypes with too few replicates, or with too low a cell count, or whose replicates covered too wide a range of measured fitnesses (as determined according to their index of dispersion). Due to differences in library diversity and measurement range between libraries, the particular cutoffs for amacGFP, cgreGFP, and ppluGFP2 differed slightly, and were selected such as to minimize the numbers of false positives and false negatives, while maximizing the total number of retained genotypes. In brief, amacGFP, cgreGFP, and ppluGFP2 genotypes were required to have, respectively: a minimum of 2, 3, and 3 replicates; cell counts over 26, 14, and 23; and indices of dispersion under 525, 575, and 1000. In each case, the final dataset showed no false negatives, i.e. wild-type proteins measured as dark (based on data from over 1000 nucleotide genotypes with synonymous mutations), while false positive rates, i.e. genotypes with chromophore mutations measured as having non-zero fluorescence, ranged from $0.24 \%$ (ppluGFP2) to $0.47 \%$ (amacGFP) to $0.71 \%$ (cgreGFP).

Of the surviving nucleotide genotypes, those with synonymous mutations coding for the same protein were merged, and assigned a fitness corresponding to the mean of the 
fitnesses of the different nucleotide genotypes, weighted by their cell count. The final dataset (Supplementary Data 3) included 35500, 26165, and 32260 unique protein sequences respectively for amacGFP, cgreGFP, and ppluGFP2.

\section{Calculation of epistasis}

Our mutant generation strategy created genotypes with an average of 3-4 mutations each. This also led to >1100 single mutants in each gene (Table S1) for which we could directly calculate their individual effects. This allowed us to determine the contribution of epistasis to the fluorescence of genotypes with multiple mutants. Epistasis was calculated as the difference between the measured fluorescence of a genotype and its expected fluorescence under the assumption that the joint effect of multiple mutations is equal to the sum of their individual effects, according to the following equality:

$$
\text { epistasis }=\text { Effect }_{\text {observed }}-\text { Effect }_{\text {expected }}=\left(F_{m}-F_{w t}\right)-\sum_{i}\left(F_{i}-F_{w t}\right) \cdot x_{i}
$$

\section{(eq. 1)}

Where $\mathrm{F}_{\mathrm{i}}, \mathrm{F}_{m} \mathrm{~F}_{\mathrm{wt}}$ are measured levels of fluorescence of a genotype with mutation $i$, of a genotype $m$ containing one or more mutations, or of the wildtype sequence, respectively, and $x_{i}=1$ when mutation $i$ is contained within the genotype $m$ and $x_{i}=0$ when it is not. In order to avoid detecting false epistasis, expected values are capped and cannot be greater than the dataset's maximum observed measurement, nor less than the minimum observed measurement. Instances of genotypes harbouring multiple mutations for which one or more of the mutations has not been observed in isolation were not included in this analysis.

\section{Protein purification}

Wildtype sequences with $\mathrm{N}$-terminal His-tags were cloned into $\mathrm{T} 7$ expression vectors via MoClo. Chemically competent BL21-DE3 (New England Biolabs) were transformed and plated on LB agar supplemented with antibiotic and 20uM IPTG, grown overnight at 30C and left at room temperature an additional day to allow extensive time for fluorescent protein maturation. Colonies from twenty $12 \times 12 \mathrm{~cm}$ plates were scraped and recovered in $40 \mathrm{ml}$ of binding buffer $(500 \mathrm{mM} \mathrm{NaCl}, 20 \mathrm{mM}$ Tris- $\mathrm{HCl}, 25 \mathrm{mM}$ imidazole, $\mathrm{pH} 8$ ), lysed in a Qsonica Q700 sonicator (20 kHz, amplitude 10, 1s on/4s off, 20 min of active sonication time), and centrifuged for 30 minutes at 20,000 g. The supernatant was recovered and incubated with rotation for one hour at $4 \mathrm{C}$ with $3 \mathrm{ml}$ of nickel-sepharose protein purification resin (Cytiva). Before use, resin was washed with 5 volumes of binding buffer, and 5 volumes of distilled water.

After incubation, the protein/resin solution was passed through an empty chromatography column (BioRad Econo-Pac), washed thrice with $20 \mathrm{ml}$ of binding buffer, then protein was recovered in $2-5 \mathrm{ml}$ of elution buffer $(500 \mathrm{mM} \mathrm{NaCl}, 20 \mathrm{mM}$ Tris- $\mathrm{HCl}, 500 \mathrm{mM}$ imidazole, $\mathrm{pH}$ 8).

\section{Crystallization, Data Collection, and Structure Determination}


AmacGFP (12 mg/mL in $20 \mathrm{mM}$ Tris- $\mathrm{HCl}$ buffer, $\mathrm{pH} 7.5)$ was crystallized at $21^{\circ} \mathrm{C}$ in $8 \%$ PEG $6 \mathrm{~K}, 3 \%$ glycerol, $0.1 \mathrm{M}$ sodium acetate, pH 5.0 supplemented with $5.0 \%$ Jeffamine ${ }^{\circledR} \mathrm{M}-600^{\circledR}$ pH 7.0 according to the Hampton Research Additive Screen protocol using the sitting drop vapor diffusion technique. Crystals grew within 1 week and were flash frozen in liquid nitrogen using mother liquor supplemented with 20\% PEG 400 as cryoprotectant.

Diffraction data were collected using the D8 Venture (Bruker AXS, Madison, WI) system that includes an Excillum D2+ MetalJet X-ray source with Helios MX optics providing Ga Ka radiation at a wavelength of $1.3418 \AA$ and a PHOTON III charge-integrating pixel array detector. Data were reduced using Proteum3 software (Bruker AXS). The crystal structures were solved by molecular replacement with MOLREP(Vagin and Teplyakov, 1997) using a avGFP mutant as a search model (PDB ID 2AWK). Model building and iterative refinement were performed with Coot(Emsley and Cowtan, 2004) and REFMAC(Murshudov et al., 1997), respectively. The final statistics of the structure are shown in Table S2. The model has been deposited into the Protein Data Bank (PDB ID 7LG4).

\section{$\Delta \Delta \mathrm{G}$ prediction and residue distance measurements}

Calculations were performed using the following structures: avGFP (PDB ID 2WUR), ppluGFP2 (PDB ID 2G3O), cgreGFP (PDB ID 2HPW), and amacGFP (PDB ID 7LG4, this study). For each structure, one (the first) chain was extracted and minimized using Rosetta Relax application (Nivón et al., 2013) with constraints to starting coordinates. The total of 50 structures were generated for each protein and the model with the lowest total score was chosen for further calculations. The GFP chromophores' (GYG and SYG) geometries were optimized in Gaussian using density functionals at the B3LYP/6-31++G(d,p) level of theory. The chromophores were treated as non-canonical amino acids (Renfrew et al., 2012). $\Delta \Delta \mathrm{G}$ calculations (Supplementary Data 4) were performed for all single mutations except for nonsense mutations, mutations in the chromophore triade, and positions that are not present in the corresponding crystal structure using Rosetta ddg_monomer application (Kellogg et al., 2011). All runs were performed with Rosetta version 3.10. Distances between amino acid residue pairs are available in Supplementary Data 5.

\section{Urea sensitivity assays}

Absorbance and fluorescence spectra were measured on Biotek SynergyH1 plate readers. For fluorescence, samples consisted of $200 \mu \mathrm{l}$ of $0.15 \mu \mathrm{M}$ purified protein in $1 \mathrm{X}$ PBS and either $0 \mathrm{M}$ or $9 \mathrm{M}$ urea, and emission was measured in $5 \mathrm{~nm}$ steps from $450 \mathrm{~nm}$ to $700 \mathrm{~nm}$ upon excitation at $420 \mathrm{~nm}$. For absorbance, samples were identical except for protein concentration, here $18.5 \mu \mathrm{M}$, and absorbance was measured in $5 \mathrm{~nm}$ steps from $300 \mathrm{~nm}$ to $700 \mathrm{~nm}$. In both cases, spectra were continuously measured for around sixty hours, at $42^{\circ} \mathrm{C}$, and a minimum of eight technical replicates were measured for each condition. All plates used were 96-well clear- and flat-bottomed plates; for fluorescence measurements, plates were also black-walled. Blanks containing elution buffer instead of protein (see: Protein purification) were also measured, and their values subtracted from those of the protein samples (Supplementary Data 6). 
To measure refolding kinetics, samples were first denatured by diluting in $9 \mathrm{M}$ urea to a final protein concentration of $0.5 \mathrm{mg} / \mathrm{ml}$ and heating at $95^{\circ} \mathrm{C}$ for five minutes. $10 \mu \mathrm{l}$ were then transferred to a 96-well flat-bottomed plate and baseline fluorescence (excitation at 485 $\mathrm{nm}$, emission at $520 \mathrm{~nm}$ ) was measured on a Biotek SynergyH1 plate reader. $200 \mu \mathrm{l}$ of $1 \mathrm{X}$ PBS was added via injection and fluorescence was immediately measured for 20 minutes at intervals of one second, or for 13.8 hours at intervals of 50 seconds.

\section{Thermosensitivity assays}

Thermal unfolding and/or aggregation of purified green fluorescent proteins was monitored by differential scanning fluorimetry (DSF), circular dichroism (CD), and differential scanning calorimetry (DSC), and fluorescence emission during heating was monitored in a Roche Lightcycler 480. Protein samples were diluted in imidazole-free elution buffer (see: Protein purification) from $20 \mathrm{mg} / \mathrm{ml}$ stocks in $500 \mathrm{mM}$ imidazole elution buffer. Raw data are available in Supplementary Data 7.

Differential scanning fluorimetry. Samples were run in triplicate on a Prometheus NT.48 (NanoTemper Technologies) machine set at 100\% excitation power. Samples consisted of $10 \mu \mathrm{l}$ of $1 \mathrm{mg} / \mathrm{ml}$ protein, heated from $20^{\circ} \mathrm{C}$ to $110^{\circ} \mathrm{C}$ at a ramp rate of $1^{\circ} \mathrm{C}$ per minute; melting temperatures for unfolding and aggregation were determined from the peaks of the first derivatives of either the $350 / 330 \mathrm{~nm}$ emission ratio or the light scattering, respectively. Although all considered GFPs contained a low content of tryptophan, the primary signal source in NanoDSF, all GFPs contained high enough tyrosine content to generate a good signal (avGFP: 1 Trp, 11 Tyr; amacGFP: 1 Trp, 10 Tyr; cgreGFP: 3 Trp, 14 Tyr; ppluGFP2: 0 Trp, 12 Tyr).

Circular dichroism. Samples consisting of $200 \mu \mathrm{l}$ of $0.1 \mathrm{mg} / \mathrm{ml}$ protein in a $1 \mathrm{~mm}$ thickness cuvette were analyzed on a Jasco J-815 CD spectropolarimeter. Initial protein spectra were measured at $30^{\circ} \mathrm{C}$, from $260 \mathrm{~nm}$ to $200 \mathrm{~nm}$, and the spectrum of protein-free buffer was subtracted; protein spectra were not measured beyond $200 \mathrm{~nm}$ as the high tension voltage in this region increased beyond $700 \mathrm{~V}$, making CD measurements unreliable. The following settings were used for spectra measurements: scanning speed of $100 \mathrm{~nm} / \mathrm{min}$; data pitch of $1 \mathrm{~nm}$; digital integration time of $2 \mathrm{~s}$ with $1 \mathrm{~nm}$ bandwidth; 10 accumulations. After measuring the initial spectra, samples were heated to $98^{\circ} \mathrm{C}$ at a rate of $1^{\circ} \mathrm{C}$ per minute, and monitored throughout at $218 \mathrm{~nm}$ (208 $\mathrm{nm}$ in the case of avGFP), a wavelength corresponding to a peak in the spectra. The full spectra were then measured again at $98^{\circ} \mathrm{C}$, under the same settings described above. The single-wavelength melting curves were fitted with a logistic curve, $f(x)=\frac{L}{1+e^{-k(x-x 0)}}$, using the Python module scipy.optimize.curve_fit, in order to obtain the melting temperature $(x 0)$ and the logistic growth rate $(k)$.

Differential scanning calorimetry. $1 \mathrm{mg} / \mathrm{ml}$ protein samples were run in duplicate on a MicroCal PEAQ-DSC (Malvern Panalytical), and measured from $20^{\circ} \mathrm{C}$ to $110^{\circ} \mathrm{C}$ at a ramp rate 
of $1^{\circ} \mathrm{C}$ per minute. Melting temperatures (temperature corresponding to the peak in specific heat capacity or $\mathrm{Cp}$ ) and enthalpies of denaturation (area under the peak) were determined automatically. DSC runs were performed by the BIC facility of CEITEC MU, Brno.

Green fluorescence emission upon heating. Fluorescence emission of purified protein samples $\left(0.1 \mathrm{mg} / \mathrm{ml}\right.$, final volume $20 \mu \mathrm{l}$ in white 96 -well plates) during heating from $20^{\circ} \mathrm{C}$ to $99^{\circ} \mathrm{C}$ at a ramp rate of $\sim 2^{\circ} \mathrm{C} / \mathrm{min}$ was measured on a Roche LightCycler 480 monitoring the SYBR-Green channel (excitation at $465 \mathrm{~nm}$, collection at $510 \mathrm{~nm}$ ). (Supplementary Data 7). Melting temperatures were determined automatically from the melting curve peak.

\section{SEC-MALS}

Size exclusion chromatography/multiangle light scattering analysis was performed on an OmniSEC system (Malvern Panalytical). Samples consisted of $0.2 \mu \mathrm{m}$-filtered, $1 \mathrm{mg} / \mathrm{ml}$ purified proteins in $20 \mathrm{mM}$ Tris $\mathrm{pH} 8,150 \mathrm{mM} \mathrm{NaCl}, 25 \mathrm{mM}$ imidazole buffer. Injection volumes were $50 \mu \mathrm{l}$. Samples were measured at $30^{\circ} \mathrm{C}$ with a flow rate of $0.7 \mathrm{~mL} / \mathrm{min}$. SEC-MALS runs were performed by the BIC facility of CEITEC MU, Brno.

\section{SDS-PAGE and Western blots}

Genome-integrated mutant libraries were plated on LB-Zeocin agar plates, colonies were recovered (pelled weight of $0.25 \mathrm{~g}$ ) and resuspended in $30 \mathrm{~mL}$ of lysis buffer (1X PBS pH 7.4, $150 \mathrm{mM} \mathrm{NaCl}$, supplemented with $50 \mu$ protease inhibitor cocktail (Sigma Aldrich, ref. P8340)). Cells were sonicated on a QSonica Q700 (20 kHz, amplitude 10, 1s on/4s off, 10 minutes of active sonication). To separate soluble and insoluble fractions, $15 \mu$ l of lysate were centrifuged for 10 minutes at 20000g, supernatant was collected and the pellet resuspended in $15 \mu \mathrm{l}$ of lysis buffer. $5 \mu \mathrm{l}$ of $4 \mathrm{X}$ Laemmli loading dye (BioRad) was added to 15 $\mu \mathrm{l}$ of either total lysate, supernatant, or resuspended pellet. Samples were boiled at $95^{\circ} \mathrm{C}$ for 5 minutes, and run in 4-20\% polyacrylamide Mini-Protean precast gels (BioRad) at 100V for one hour. The Protein Precision Plus Standard (BioRad) was used as a molecular weight marker. Gels were stained with a colloidal Coomassie dye, ReadyBlue (Sigma-Aldrich), overnight at room temperature.

For Western blot, gels were transferred to PVDF membranes (BioRad) using a Trans-Blot Turbo Transfer system (BioRad), blocked with EveryBlot blocking buffer (BioRad) for 15 minutes at room temperature, and incubated overnight at $4^{\circ} \mathrm{C}$ with a mouse monoclonal anti-His-tag primary antibody (Abcam, ref. ab18184) diluted 1:1000. Membranes were washed in 1X PBS/0.05\% Tween-20 (five 5-minute washes), incubated for two hours at room temperature with 1:1000 anti-mouse HRP secondary antibody (Cell Signal, ref. \#7076), washed, and incubated with SuperSignal West Pico-Plus ECL substrate and imaged on a ChemiDoc MP system (BioRad).

\section{Experimental testing of predictions}


Coding sequences for neural network-generated genotypes (Supplementary Data 8) were ordered as dsDNA from Twist Biosciences, flanked by BsaI restriction sites for MoClo insertion into destination vectors. GFP sequences were cloned into a medium/low-copy vector conferring zeocin resistance, under a constitutive T5 promoter and lambda t0 terminator, and transformed into XL10-Gold chemically competent cells. Cells were plated on LB-zeocin agar supplemented with ink (1\%) to improve fluorescence visualization. Colonies of each construct were picked and sent for Sanger sequence confirmation (Microsynth). Photographs of plates were taken with a Canon EOS 600D SLR camera. For comparison of fluorescence of different genotypes, photographs of plates containing streaks of all wild-types and mutants were photographed under identical conditions (aperture 2.8, ISO 100, 0.8 seconds exposure time), images were converted to 8 bit in FIJI and median pixel values were determined for each streak. Brightness, contrast, or other image parameters were not altered, and none of the images used contained any saturated pixels.

\section{Sequence and evolutionary analysis}

A total of 68 GFP sequences with confirmed emission in the green spectrum were selected using available information from the literature. These sequences (Supplementary Data 9) were used in the analysis of the fraction of deleterious amino acids in one of the four wildtype sequences that were neutral in another genotype (Figure 3) and in determining extant amino acid states (Figure S12). To calculate the rate of evolution of mutations that were used in successful predictions of distant functional GFPs we aligned these 68 amino acid sequences with muscle (Edgar, 2004), trimmed the alignment and made a phylogenetic reconstruction with MrBayes (Ronquist et al., 2012), reconstructed the ancestral state of a non-trimmel codon alignments and calculated the ds per each branch of the tree by codeml (Yang, 2007). Finally, we compared the rate of evolution between the two amino acid states (the one found in the wildtype and the other corresponding to an amino acid state used in at least one of the successful neural network predictions) to the rate of synonymous evolution (ds) at the same branches (Supplementary Data 10).

\section{Modelling the fitness landscape of GFPs with neural networks}

For all 4 fitness landscapes, the log10-transformed fluorescence (fluorescence for short) is a bimodal distribution of two normals with very little overlap. One mode corresponds to non-functional genotypes while the other mode corresponds to functional genotypes of near wild-type fluorescence levels. In each dataset, genotypes associated with negative fluorescence have been excluded to ensure that the four distributions cover similar ranges. The genotype-phenotype datasets were split randomly into training, validation and test sets (60\%, 20\% 20\%). To evaluate the complexity of the genotype-phenotype relationship in the four landscapes, we trained neural networks of increasing complexity on one-hot encoded protein sequences with the task of predicting fluorescence level. One-hot is a binary encoding that represents which amino acid is present or absent for each position in a sequence. All models were built using Keras (Chollet, 2015). Model goodness was 
measured as the coefficient of determination between known and predicted fluorescence values associated with genotypes in the validation set.

For each dataset, a linear model defined as a neural network containing only an input layer and one layer of a single neuron with linear activation was trained for a maximum of 30 epochs with the objective to minimise the loss as defined by the mean squared error (MSE) between actual and predicted fluorescence levels. Overfitting was prevented by monitoring the validation loss with a patience of 10 epochs. These baseline models output a simple estimate of the fluorescence level, the fitness potential, associated with each genotype. It is simply the summed contribution of mutations assigned individual weights. Models with a sigmoid output node were obtained by adding a single neuron with sigmoid activation function to these architectures and retraining on the training set.

In order to capture and visualise the non-trivial functions transforming the fitness potential into the predicted fluorescence, we trained models containing an input layer, a hidden layer with one linear node, computing the fitness potential, a second hidden layer of 10 sigmoid nodes and one linear output node outputting the fluorescence. The output subnetwork of 10 sigmoids allows the models to approximate a wide variety of sophisticated functions. For each genotype in the validation set, we computed the fitness potential as the output of the first hidden layer, and the predicted fluorescence. The output subnetworks were able to accurately capture the functions transforming the fitness potential into fluorescence level, revealing non-trivial sigmoid functions (Figure S14d).

Optimisation of artificial neural nets was performed using a random grid search approach. Tested architectures contained one input layer, one hidden layer with linear nodes, a second hidden layer with sigmoid nodes, and one linear output node. The two hidden layers were built with random numbers of neurons, picked from 1 to 10, 20, 50, 100, 200. The models also contained one Monte Carlo dropout layer after each hidden layer (rate=0.1, training=True), but not after the output node (Figure 4a). MC Dropout layers present the double advantage of preventing overfitting and allowing the model to predict the fluorescence of each genotype with uncertainty estimates(Hinton et al., 2012; Srivastava et al., 2014). Each architecture was trained for 10 epochs and the architecture with the smallest loss (MSE) on the validation set was selected for further training to a maximum of 30 epochs. To ensure fair training of the optimised models, the training set was filtered to exclude genotypes containing mutations present in less than 10 distinct genotypes. Since removing a genotype with a rare mutation also decreases the number of occurrences of the other mutations this genotype may contain, the filtration process was repeated until all mutations in the training set were present in at least 10 genotypes and therefore no further genotype had to be removed. To ensure fair scoring of the optimised models, the validation set was filtered to remove genotypes that contained mutations absent from the training set. This ensures the neural networks are trained on enough examples for each mutation, and the model's final score is not underestimated due to poorly trained mutations present 
in the validation set. These models were then used as part of a genetic algorithm to predict distant functional genotypes.

For each gene, an additional model with 10, 100 and 1 leaky ReLU nodes was trained and validated independently on $90 \%$ and $10 \%$ of the dataset respectively for a maximum of 500 epochs, minimising MSE loss. Overfitting was prevented by monitoring the validation loss with a patience of 10 epochs. Coefficients of determination were 0.710, 0.740, and 0.810 for amacGFP, cgreGFP and ppluGFP2 respectively. These models were used to filter genetic algorithm predictions a posteriori with an independent predictor.

\section{Prediction of distant functional genotypes}

Prediction of distant functional genotypes was performed using a genetic algorithm approach. An initial population of 50 wild-type genotypes is initialised. At each generation the genotypes were shuffled and half of the population was put aside to remain untouched. The other half undergoes crossing-overs and mutations. Crossing-overs were performed randomly along pairs of genotypes without gene conversion. Crossing-overs had a 0.7 probability of occuring in each couple of sequences and the number of crossing-overs was chosen randomly in the range of 0 to 5. Resulting genotypes (some of which may not have been crossed) underwent a mutagenesis step. Mutations were picked from a random pool containing mutated states but also wild-type states to allow the algorithm a chance to revert evolutionary dead-ends. If the targeted number of mutations defined by the user was exceeded in a genotype, the algorithm removed one previously added mutation from the genotype, allowing heavily mutated sequences to gradually bounce back to the target value. Per amino-acid mutation probabilities were defined empirically in the range of 0.01-0.015. After crossing-overs and mutations, the new genotypes were added to the rest of the population.

Mutations available to the genetic algorithm were selected using the following approach: we excluded mutations that had been seen by the model in less than 10 distinct genotypes during training of the optimised neural nets. From the remaining mutations, we kept those for which we could find in the dataset both genotypes that had those mutations and identical counterparts or "background" genotypes without these mutations. If at least 5 pairs of corresponding genotypes could be found, the impact of the mutation was computed by subtracting the fluorescence levels of the genotypes without the mutation to the fluorescence levels of the genotypes that contain the mutation and taking the median. Last, only mutations with a median impact greater or equal to $-0.1 \log 10$ fluorescence units were fed into the genetic algorithm. In short, this approach excludes severely deleterious mutations from the genetic algorithm.

Finally, to ensure predicted genotypes did not converge to "known" functional genotypes, the pool of usable mutations was enriched in mutations that do not lead to states observed in natural GFP sequences. To do so, all sequences were scraped from FPbase (June 2020), filtered to keep green natural ones and aligned on a profile obtained from the wild-type 
sequence of amacGFP, avGFP, cgreGFP and ppluGFP2. We then applied in the pool of mutations available to the genetic algorithm, a ratio of 0.6 in favor of mutations that could not be found in natural GFPs.

After the crossing-over and mutation steps, the genotypes were one-hot encoded and their fluorescence level was updated by taking the median of 20 outcomes computed by the optimised neural network. The genotypes were sorted by descending fluorescence and only top genotypes were kept to maintain a constant population size. This process was repeated for several generations. Crossing-over and mutation rates, number of generations, and the ratio between mutated or wild-type available states were adjusted empirically to allow most genotypes in the population to reach the desired number of mutations while evolving to improved fluorescence levels. Notably, the algorithm was stopped a few generations after the median of predicted fluorescence levels in the population reached a plateau. This was to ensure the algorithm selects the best performing genotypes at the desired number of mutations while maintaining sequence diversity in the population. The entire algorithm was repeated until all unique mutations in the pool had been sampled, with a minimum of 10 replicates.

The resulting predictions were filtered to keep unique genotypes that contained the required number of mutations and whose fluorescence \pm standard deviation as computed by the optimised and the posteriori neural networks was greater than fluorescence level of their wild-type counterpart. Finally we used cd-hit(Fu et al., 2012; Li and Godzik, 2006) iteratively with a similarity threshold decreasing at each iteration until the remaining set of candidate sequences could be separated into the desired number of clusters. One representative sequence per cluster was picked for experimental validation. 
Table S1. Selected statistics of genotypes at different divergence from five GFP sequences

\begin{tabular}{|c|c|c|c|c|c|c|c|c|}
\hline & Number of mutations & 1 & 2 & 3 & 4 & 5 & 6 & 7 \\
\hline amacGFP & $\begin{array}{l}\text { Number of genotypes } \\
\text { Median fluorescence } \\
\text { Fraction wildtype-like* } \\
\text { Fraction dark**}\end{array}$ & $\begin{array}{l}1214 \\
3.94 \\
63.7 \% \\
9.0 \%\end{array}$ & $\begin{array}{l}10439 \\
3.9 \\
44.7 \% \\
11.7 \%\end{array}$ & $\begin{array}{l}6389 \\
3.85 \\
31.2 \% \\
19.6 \%\end{array}$ & $\begin{array}{l}3077 \\
3.75 \\
21.6 \% \\
27.9 \%\end{array}$ & $\begin{array}{l}1269 \\
3.44 \\
15.2 \% \\
38.4 \%\end{array}$ & $\begin{array}{l}511 \\
2.82 \\
8.6 \% \\
55.0 \%\end{array}$ & $\begin{array}{l}188 \\
2.74 \\
8.5 \% \\
63.3 \%\end{array}$ \\
\hline $\begin{array}{l}\text { amacGFP: } \\
\text { V14L }\end{array}$ & $\begin{array}{l}\text { Number of genotypes } \\
\text { Median fluorescence } \\
\text { Fraction wildtype-like* } \\
\text { Fraction dark**}\end{array}$ & $\begin{array}{l}1068 \\
3.93 \\
65.0 \% \\
5.8 \%\end{array}$ & $\begin{array}{l}5621 \\
3.89 \\
46.3 \% \\
10.7 \%\end{array}$ & $\begin{array}{l}3010 \\
3.85 \\
33.0 \% \\
16.8 \%\end{array}$ & $\begin{array}{l}1313 \\
3.78 \\
24.1 \% \\
27.3 \%\end{array}$ & $\begin{array}{l}534 \\
3.46 \\
16.7 \% \\
42.1 \%\end{array}$ & $\begin{array}{l}207 \\
2.83 \\
9.7 \% \\
52.3 \%\end{array}$ & $\begin{array}{l}84 \\
2.72 \\
4.8 \% \\
67.9 \%\end{array}$ \\
\hline cgreGFP & $\begin{array}{l}\text { Number of genotypes } \\
\text { Median fluorescence } \\
\text { Fraction WT-like* } \\
\text { Fraction dark**}\end{array}$ & $\begin{array}{l}1188 \\
4.43 \\
44.4 \% \\
14.5 \%\end{array}$ & $\begin{array}{l}10347 \\
4.36 \\
24.3 \% \\
23.7 \%\end{array}$ & $\begin{array}{l}6567 \\
3.62 \\
11.4 \% \\
45.2 \%\end{array}$ & $\begin{array}{l}3666 \\
2.79 \\
5.7 \% \\
65.5 \%\end{array}$ & $\begin{array}{l}1959 \\
2.77 \\
2.0 \% \\
81.4 \%\end{array}$ & $\begin{array}{l}1061 \\
2.77 \\
0.9 \% \\
87.6 \%\end{array}$ & $\begin{array}{l}546 \\
2.77 \\
0.2 \% \\
92.3 \%\end{array}$ \\
\hline ppluGFP2 & $\begin{array}{l}\text { Number of genotypes } \\
\text { Median fluorescence } \\
\text { Fraction wildtype-like* } \\
\text { Fraction dark**}\end{array}$ & $\begin{array}{l}1163 \\
4.2 \\
66.1 \% \\
4.4 \%\end{array}$ & $\begin{array}{l}16134 \\
4.16 \\
43.8 \% \\
10.2 \%\end{array}$ & $\begin{array}{l}8920 \\
4.1 \\
29.7 \% \\
18.7 \%\end{array}$ & $\begin{array}{l}3710 \\
3.96 \\
19.6 \% \\
29.8 \%\end{array}$ & $\begin{array}{l}1370 \\
3.67 \\
16.0 \% \\
38.8 \%\end{array}$ & $\begin{array}{l}456 \\
3.32 \\
17.1 \% \\
44.5 \%\end{array}$ & $\begin{array}{l}186 \\
2.86 \\
11.3 \% \\
60.2 \%\end{array}$ \\
\hline avGFP & $\begin{array}{l}\text { Number of genotypes } \\
\text { Median fluorescence } \\
\text { Fraction wildtype-like* } \\
\text { Fraction dark**}\end{array}$ & $\begin{array}{l}1114 \\
3.64 \\
68.9 \% \\
9.4 \%\end{array}$ & $\begin{array}{l}13010 \\
3.59 \\
55.5 \% \\
12.4 \%\end{array}$ & $\begin{array}{l}12683 \\
3.49 \\
39.1 \% \\
27.0 \%\end{array}$ & $\begin{array}{l}9759 \\
3.14 \\
23.3 \% \\
47.9 \%\end{array}$ & $\begin{array}{l}7215 \\
1.53 \\
13.1 \% \\
68.4 \%\end{array}$ & $\begin{array}{l}4643 \\
1.43 \\
6.6 \% \\
83.0 \%\end{array}$ & $\begin{array}{l}2783 \\
1.36 \\
2.3 \% \\
92.0 \%\end{array}$ \\
\hline
\end{tabular}

*Fraction wildtype-like refers to the fraction of genotypes displaying fluorescence levels within two standard deviations of the wildtype, or brighter.

**Fraction dark refers to the fraction of fully non-functional genotypes (i.e. with fluorescence values falling within the darkest FACS gate). Remaining genotypes not accounted for in these two categories displayed a range of intermediate fluorescence levels. 
Table S2. Data Collection and Refinement Statistics

\begin{tabular}{|c|c|}
\hline & amacGFP (PDB ID 7LG4) \\
\hline Wavelength $(\AA)$ & 1.3418 \\
\hline Space group & $\mathrm{P} 12,1$ \\
\hline Unit cell dimensions & $\begin{array}{l}\mathrm{a}=34.089 \\
\mathrm{~b}=47.488 \\
\mathrm{c}=69.294 \\
\alpha=90^{\circ} \\
\beta=102.01^{\circ} \\
\gamma=90^{\circ}\end{array}$ \\
\hline Resolution range $(\AA)$ & $22.59-1.81(1.91-1.81)$ \\
\hline Total no. of reflections & 151,191 \\
\hline Unique reflections & $19,894(2,932)$ \\
\hline Completeness (\%) & $99.8(99.5)$ \\
\hline Multiplicity & $7.59(4.69)$ \\
\hline Mean I / $\sigma(\mathrm{I})$ & $9.36(1.93)$ \\
\hline $\mathrm{CC}_{1 / 2}$ & $0.996(0.624)$ \\
\hline \multicolumn{2}{|l|}{ Refinement Statistics } \\
\hline Rwork/Rfree(\%) & $18.17 / 22.75$ \\
\hline Average B factor $\left(\AA^{2}\right)$ & 20.14 \\
\hline Total no. of atoms & 2,051 \\
\hline Protein atoms & 1,863 \\
\hline Water molecules & 134 \\
\hline Protein residues & 228 \\
\hline Bond angles $\left(^{\circ}\right)$ & 1.91 \\
\hline Bond length $(\AA)$ & 0.014 \\
\hline Ramachandran: favored/allowed (\%) & $98.65 / 1.35$ \\
\hline Clashscore & 5.77 \\
\hline
\end{tabular}


bioRxiv preprint doi: https://doi.org/10.1101/2021.12.08.471728; this version posted December 9, 2021. The copyright holder for this preprint (which was not certified by peer review) is the author/funder, who has granted bioRxiv a license to display the preprint in perpetuity. It is made available under aCC-BY 4.0 International license.

Table S3. Primers used for PCRs in sample preparation for NGS

\begin{tabular}{|c|c|c|c|}
\hline & Primer purpose & Forward & Reverse \\
\hline \#1 & $\begin{array}{l}\text { dsDNA oligo filler for } \\
\text { circularization }\end{array}$ & $\begin{array}{c}\text { ATAAAGGTCTCAAGGTCGCCCTGAG } \\
\text { CCGCTACTACCAATGAGAGACCAAT } \\
\text { AT }\end{array}$ & $\begin{array}{c}\text { ATATTGGTCTCTCATTGGTAGTAGCGGCTCA } \\
\text { GGGCGACCTTGAGACCTTTAT }\end{array}$ \\
\hline$\# 2$ & $\begin{array}{l}\text { First PCR of N-terminal } \\
\text { amacGFP, for MiSeq }\end{array}$ & $\begin{array}{c}\text { CCCTACACGACGCTCTTCCGATCTN } \\
\text { NNNGATGATGAGCGGCGCCTAGGAA } \\
\text { CA } \\
\text { or } \\
\text { CCCTACACGACGCTCTTCCGATCTN } \\
\text { NNGATGATGAGCGGCGCCTAGGAAC } \\
\text { A } \\
\text { or } \\
\text { CCCTACACGACGCTCTTCCGATCTN } \\
\mathbf{N G A T G A T G A G C G G C G C C T A G G A A C A ~}\end{array}$ & $\begin{array}{c}\text { GTGACTGGAGTTCAGACGTGTGCTCTTCCGA } \\
\text { TCNNGTCCATGCCCTTCAGCTCGATGCG } \\
\text { or } \\
\text { GTGACTGGAGTTCAGACGTGTGCTCTTCCGA } \\
\text { TCNNNGTCCATGCCCTTCAGCTCGATGCG } \\
\text { or } \\
\text { GTGACTGGAGTTCAGACGTGTGCTCTTCCGA } \\
\text { TCNNNNGTCCATGCCCTTCAGCTCGATGCG }\end{array}$ \\
\hline \#3 & $\begin{array}{l}\text { First PCR of C-terminal } \\
\text { amacGFP, for MiSeq }\end{array}$ & $\begin{array}{c}\text { CCCTACACGACGCTCTTCCGATCTN } \\
\mathbf{N G T G A A G T T C G A G G G C G A C A C A C T G ~} \\
\text { or } \\
\text { CCCTACACGACGCTCTTCCGATCTN } \\
\text { NNGTGAAGTTCGAGGGCGACACACT } \\
\text { G } \\
\text { or } \\
\text { CCCTACACGACGCTCTTCCGATCTN } \\
\text { NNNGTGAAGTCGAGGGCGACACAC } \\
\text { TG }\end{array}$ & $\begin{array}{c}\text { GTGACTGGAGTTCAGACGTGTGCTCTTCCGA } \\
\text { TCNNNNACCACAGAGTACTTCGTGGTCTCA } \\
\text { or } \\
\text { GTGACTGGAGTTCAGACGTGTGCTCTTCCGA } \\
\text { TCNNNACCACAGAGTACTTCGTGGTCTCA } \\
\text { or } \\
\text { GTGACTGGAGTTCAGACGTGTGCTCTTCCGA } \\
\text { TCNNACCACAGAGTACTTCGTGGTCTCA }\end{array}$ \\
\hline$\# 4$ & $\begin{array}{l}\text { First PCR of N-terminal } \\
\text { cgreGFP, for MiSeq }\end{array}$ & See Forward \#2 & $\begin{array}{c}\text { GTGACTGGAGTTCAGACGTGTGCTCTTCCGA } \\
\text { TCNNNNTCCCAGGATGTTGCCGTTTGACT } \\
\text { or } \\
\text { GTGACTGGAGTTCAGACGTGTGCTCTTCCGA } \\
\text { TCNNNTCCCCAGGATGTTGCCGTTTGACT } \\
\text { or } \\
\text { GTGACTGGAGTTCAGACGTGTGCTCTTCCGA } \\
\text { TCNNTCCCCAGGATGTTGCCGTTTGACT }\end{array}$ \\
\hline$\# 5$ & $\begin{array}{l}\text { First PCR of C-terminal } \\
\text { cgreGFP, for MiSeq }\end{array}$ & $\begin{array}{c}\text { CCCTACACGACGCTCTTCCGATCTN } \\
\text { NGTTCGACAATGACGGCCAGTACGA } \\
\text { or } \\
\text { CCCTACACGACGCTCTTCCGATCTN } \\
\text { NNGTTCGACAATGACGGCCAGTACG } \\
\text { A } \\
\text { or } \\
\text { CCCTACACGACGCTCTTCCGATCTN } \\
\text { NNNGTTCGACAATGACGGCCAGTAC } \\
\text { GA }\end{array}$ & See Reverse \#3 \\
\hline$\# 6$ & $\begin{array}{l}\text { First PCR of N-terminal } \\
\text { ppluGFP2, for MiSeq }\end{array}$ & See Forward \#2 & $\begin{array}{c}\text { GTGACTGGAGTTCAGACGTGTGCTCTTCCGA } \\
\text { TCNNNNAGCCTGTGCCCACTACCTTGAAGTC } \\
\text { or } \\
\text { GTGACTGGAGTTCAGACGTGTGCTCTTCCGA } \\
\text { TCNNNAGCCTGTGCCCACTACCTTGAAGTC } \\
\text { or } \\
\text { GTGACTGGAGTTCAGACGTGTGCTCTTCCGA } \\
\text { TCNNAGCCTGTGCCCACTACCTTGAAGTC }\end{array}$ \\
\hline$\# 7$ & $\begin{array}{l}\text { First PCR of C-terminal } \\
\text { ppluGFP2, for MiSeq }\end{array}$ & $\begin{array}{c}\text { CCCTACACGACGCTCTTCCGATCTN } \\
\text { NGCATTGAAAAGTACGAGGACGGCG } \\
\text { or }\end{array}$ & See Reverse \#3 \\
\hline
\end{tabular}


bioRxiv preprint doi: https://doi.org/10.1101/2021.12.08.471728; this version posted December 9,2021. The copyright holder for this preprint (which was not certified by peer review) is the author/funder, who has granted bioRxiv a license to display the preprint in perpetuity. It is made available under aCC-BY 4.0 International license.

\begin{tabular}{|c|c|c|c|}
\hline & & $\begin{array}{c}\text { CCСTACACGACGCTCTTCCGATCTN } \\
\text { NNGCATTGAAAAGTACGAGGACGGC } \\
\text { G } \\
\text { or } \\
\text { CCCTACACGACGCTCTTCCGATCTN } \\
\text { NNNGCATTGAAAAGTACGAGGACGG } \\
\text { CG }\end{array}$ & \\
\hline \#8 & First PCR for HiSeq, all genes & See Forward \#2 & $\begin{array}{l}\text { GTGACTGGAGTTCAGACGTGTGCTCTTCCGA } \\
\text { TCNNAACCGCCGAGGTCAGTTTCGCC }\end{array}$ \\
\hline \#9 & $\begin{array}{l}\text { Second PCR, MiSeq and } \\
\text { Hiseq: adding full TruSeq } \\
\text { adapters, universal (forward) } \\
\text { and indexed (reverse) }\end{array}$ & $\begin{array}{c}\text { AATGATACGGCGACCACCGAGATCT } \\
\text { ACACTCTTTCCCTACACGACGCTCT } \\
\text { TCCGATCT }\end{array}$ & 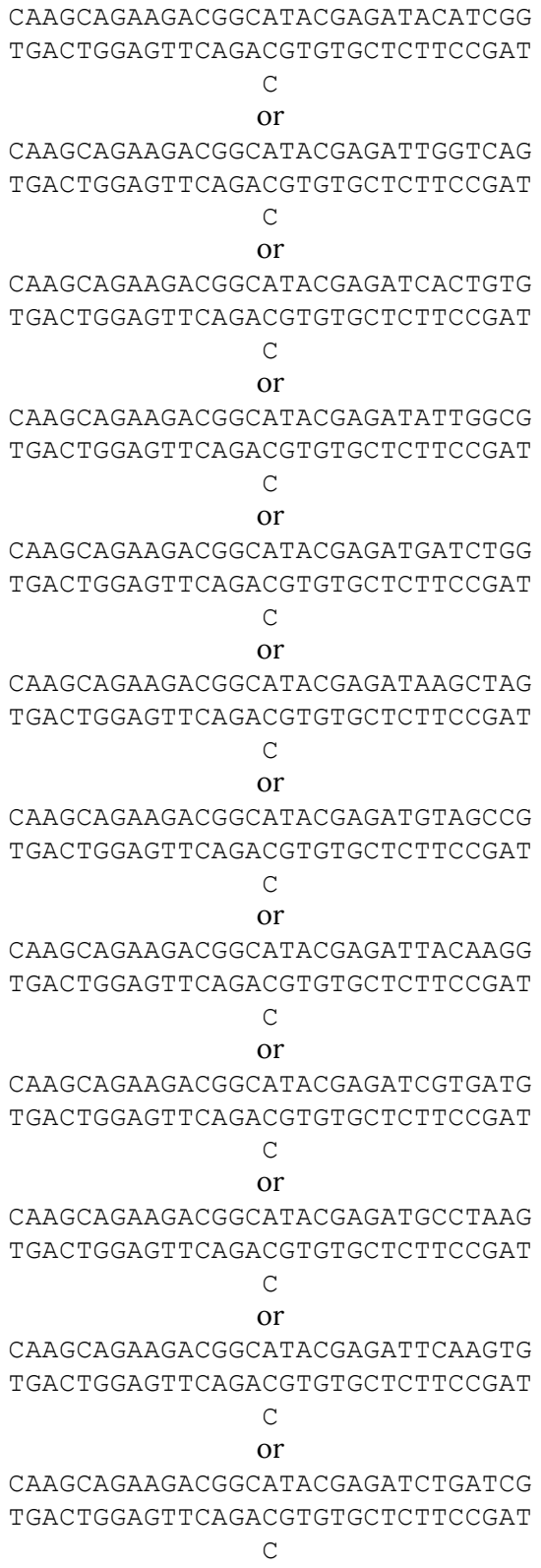 \\
\hline
\end{tabular}

Table S4. Benchling links to genome integration constructs. 
bioRxiv preprint doi: https://doi.org/10.1101/2021.12.08.471728; this version posted December 9,2021 . The copyright holder for this preprint (which was not certified by peer review) is the author/funder, who has granted bioRxiv a license to display the preprint in perpetuity. It is made available under aCC-BY 4.0 International license.

\begin{tabular}{|l|l|}
\hline Name & Link \\
\hline amacGFP & https://benchling.com/s/seq-AEhUmO6f9dWC2uxMNXxO \\
\hline cgreGFP & https://benchling.com/s/seq-t47EdbWNoBftyZYQtCQs \\
\hline ppluGFP2 & https://benchling.com/s/seq-CEgJq6FMBm5yzd5oDcKm \\
\hline
\end{tabular}




\section{Supplementary Data file descriptions}

\section{Supplementary Data 1}

Absolute values for the borders between gates in the green channel during sorting, for all genes and machines, and the corrections applied to match values between the machines.

\section{Supplementary Data 2}

Dataframes containing the distribution across gates of all primary-secondary barcode combinations, along with their fitted fitness values (see Methods). Data are not filtered according to cell count, number of replicates, etc. One dataframe per gene and machine.

\section{Supplementary Data 3}

Dataframes linking nucleotide or protein genotypes to their measured fluorescence level (see Methods). Mutations in genotypes are labeled in the format AiB, where A is the original wildtype state, $B$ is the mutated state, and i is the position (counting starts from Methionine $=0$ ). In the nucleotide dataset, 'n_replicates' refers to the combined number of distinct barcodes representing a genotype and machines it was measured on. In the amino acid dataset, 'n_replicates' refers to the number of synonymous nucleotide sequences measured for each protein sequence. Nucleotide genotypes and amino acid genotypes are on separate tabs in the file.

\section{Supplementary Data 4}

Table containing ddG predictions for single mutations in avGFP, amacGFP, amacGFP:V12L, cgreGFP, and ppluGFP2. Residue positions are labeled starting from 0 (methionine).

\section{Supplementary Data 5}

Dataframes containing the minimum physical distance between pairs of residues inside the 3D GFP structures, in Angstroms. Row and column indices represent the residue position within the protein, starting from 0 for the initial methionine. Matrices for different proteins are included in different tabs in the file.

\section{Supplementary Data 6}

Table containing absorbance values (from 300 to $700 \mathrm{~nm}$ ) and fluorescence emission values (from 450nm to 700nm, upon 420nm excitation) for all genes, in 9M urea and PBS, measured on a plate reader at multiple consecutive time points. Blank control values are already subtracted. Absorbance and fluorescence data are listed on separate tabs.

\section{Supplementary Data 7}

Raw data from differential scanning fluorimetry and calorimetry, circular dichroism, and qPCR melting curves. 


\section{Supplementary Data 8}

Coding sequences for neural network-generated genotypes, and their predicted and observed levels of fluorescence.

\section{Supplementary Data 9}

Table of over 70 documented natural fluorescent proteins used during analyses, including name, species, sequence, original reference and, where possible, accession numbers and measured excitation/emission peaks.

\section{Supplementary Data 10}

Estimated rates of evolution of amino acid states used in prediction of novel GFP sequences on each branch of the phylogeny of extant GFPs. 


\section{References}

Alley EC, Khimulya G, Biswas S, AlQuraishi M, Church GM. 2019. Unified rational protein engineering with sequence-based deep representation learning. Nat Methods 16:1315-1322.

Armougom F, Moretti S, Poirot O, Audic S, Dumas P, Schaeli B, Keduas V, Notredame C. 2006. Expresso: automatic incorporation of structural information in multiple sequence alignments using 3D-Coffee. Nucleic Acids Res 34:W604-8.

Bank C, Hietpas RT, Jensen JD, Bolon DNA. 2015. A systematic survey of an intragenic epistatic landscape. Mol Biol Evol 32:229-238.

Bassalo MC, Garst AD, Halweg-Edwards AL, Grau WC, Domaille DW, Mutalik VK, Arkin AP, Gill RT. 2016. Rapid and Efficient One-Step Metabolic Pathway Integration inE. coli. ACS Synthetic Biology. doi:10.1021/acssynbio.5b00187

Bershtein S, Segal M, Bekerman R, Tokuriki N, Tawfik DS. 2006. Robustness-epistasis link shapes the fitness landscape of a randomly drifting protein. Nature 444:929-932.

Biswas S, Khimulya G, Alley EC, Esvelt KM, Church GM. 2021. Low-N protein engineering with data-efficient deep learning. Nat Methods 18:389-396.

Biswas S, Khimulya G, Alley EC, Esvelt KM, Church GM. 2020. Low-N protein engineering with data-efficient deep learning. Cold Spring Harbor Laboratory. doi:10.1101/2020.01.23.917682

Biswas S, Kuznetsov G, Ogden PJ, Conway NJ. 2018. Toward machine-guided design of proteins. bioRxiv.

Bryant DH, Bashir A, Sinai S, Jain NK, Ogden PJ, Riley PF, Church GM, Colwell LJ, Kelsic ED. 2021a. Deep diversification of an AAV capsid protein by machine learning. Nat Biotechnol. doi:10.1038/s41587-020-00793-4

Bryant DH, Bashir A, Sinai S, Jain NK, Ogden PJ, Riley PF, Church GM, Colwell LJ, Kelsic ED. 2021b. Deep diversification of an AAV capsid protein by machine learning. Nat Biotechnol 39:691-696.

Canale AS, Cote-Hammarlof PA, Flynn JM, Bolon DN. 2018. Evolutionary mechanisms studied through protein fitness landscapes. Curr Opin Struct Biol 48:141-148.

Chan YH, Venev SV, Zeldovich KB, Matthews CR. 2017. Correlation of fitness landscapes from three orthologous TIM barrels originates from sequence and structure constraints. Nat Commun 8:14614.

Chollet F. 2015. Keras. GitHub repository.

Codoñer FM, Darós J-A, Solé RV, Elena SF. 2006. The fittest versus the flattest: experimental confirmation of the quasispecies effect with subviral pathogens. PLoS Pathog 2:e136.

Creighton TE, Creighton TE. 1993. Proteins: Structures and Molecular Properties.

de Visser JAGM, Hermisson J, Wagner GP, Ancel Meyers L, Bagheri-Chaichian H, Blanchard JL, Chao L, Cheverud JM, Elena SF, Fontana W, Gibson G, Hansen TF, Krakauer D, Lewontin RC, Ofria C, Rice SH, von Dassow G, Wagner A, Whitlock MC. 2003. Perspective: Evolution and detection of genetic robustness. Evolution 57:1959-1972.

de Visser JAGM, Krug J. 2014. Empirical fitness landscapes and the predictability of evolution. Nat Rev Genet 15:480-490.

Draghi JA, Parsons TL, Wagner GP, Plotkin JB. 2010. Mutational robustness can facilitate adaptation. Nature 463:353-355.

Echave J, Wilke CO. 2017. Biophysical Models of Protein Evolution: Understanding the 
Patterns of Evolutionary Sequence Divergence. Annu Rev Biophys 46:85-103.

Edgar RC. 2004. MUSCLE: multiple sequence alignment with high accuracy and high throughput. Nucleic Acids Res 32:1792-1797.

Emsley P, Cowtan K. 2004. Coot: model-building tools for molecular graphics. Acta Crystallogr D Biol Crystallogr 60:2126-2132.

Ferretti L, Weinreich D, Tajima F, Achaz G. 2018. Evolutionary constraints in fitness landscapes. Heredity 121:466-481.

Fragata I, Blanckaert A, Dias Louro MA, Liberles DA, Bank C. 2019. Evolution in the light of fitness landscape theory. Trends Ecol Evol 34:69-82.

Fu L, Niu B, Zhu Z, Wu S, Li W. 2012. CD-HIT: accelerated for clustering the next-generation sequencing data. Bioinformatics 28:3150-3152.

Gong LI, Suchard MA, Bloom JD. 2013. Stability-mediated epistasis constrains the evolution of an influenza protein. Elife 2:e00631.

Haddox HK, Dingens AS, Hilton SK, Overbaugh J, Bloom JD. 2018. Mapping mutational effects along the evolutionary landscape of HIV envelope. Elife 7. doi:10.7554/eLife.34420

Hartman EC, Tullman-Ercek D. 2019. Learning from protein fitness landscapes: a review of mutability, epistasis, and evolution. Current Opinion in Systems Biology 14:25-31.

Hinton GE, Srivastava N, Krizhevsky A, Sutskever I, Salakhutdinov RR. 2012. Improving neural networks by preventing co-adaptation of feature detectors. arXiv [csNE].

Hirabayashi J, Arai R. 2019. Lectin engineering: the possible and the actual. Interface Focus 9:20180068.

Jacquier H, Birgy A, Le Nagard H, Mechulam Y, Schmitt E, Glodt J, Bercot B, Petit E, Poulain J, Barnaud G, Gros P-A, Tenaillon O. 2013. Capturing the mutational landscape of the beta-lactamase TEM-1. Proc Natl Acad Sci U S A 110:13067-13072.

Johnson MS, Martsul A, Kryazhimskiy S, Desai MM. 2019. Higher-fitness yeast genotypes are less robust to deleterious mutations. Science 366:490-493.

Keefe AD, Szostak JW. 2001. Functional proteins from a random-sequence library. Nature 410:715-718.

Kellogg EH, Leaver-Fay A, Baker D. 2011. Role of conformational sampling in computing mutation-induced changes in protein structure and stability. Proteins: Struct Funct Bioinf 79:830-838.

Kemble H, Nghe P, Tenaillon O. 2019. Recent insights into the genotype-phenotype relationship from massively parallel genetic assays. Evol Appl 12:1721-1742.

Kimura M, Crow JF. 1978. Effect of overall phenotypic selection on genetic change at individual loci. Proc Natl Acad Sci U S A 75:6168-6171.

Klug A, Park S-C, Krug J. 2019. Recombination and mutational robustness in neutral fitness landscapes. PLoS Comput Biol 15:e1006884.

Kondrashov DA, Kondrashov FA. 2015. Topological features of rugged fitness landscapes in sequence space. Trends Genet 31:24-33.

Kumar A, Natarajan C, Moriyama H, Witt CC, Weber RE, Fago A, Storz JF. 2017. Stability-Mediated Epistasis Restricts Accessible Mutational Pathways in the Functional Evolution of Avian Hemoglobin. Mol Biol Evol 34:1240-1251.

Kuo S-T, Jahn R-L, Cheng Y-J, Chen Y-L, Lee Y-J, Hollfelder F, Wen J-D, Chou H-HD. 2020. Global fitness landscapes of the Shine-Dalgarno sequence. Genome Res 30:711-723.

Kurahashi R, Sano S, Takano K. 2018. Protein Evolution is Potentially Governed by Protein Stability: Directed Evolution of an Esterase from the Hyperthermophilic Archaeon Sulfolobus tokodaii. J Mol Evol 86:283-292. 
Lässig M, Mustonen V, Walczak AM. 2017. Predicting evolution. Nat Ecol Evol 1:77.

Lee JM, Huddleston J, Doud MB, Hooper KA, Wu NC, Bedford T, Bloom JD. 2018. Deep mutational scanning of hemagglutinin helps predict evolutionary fates of human H3N2 influenza variants. Proc Natl Acad Sci U S A 115:E8276-E8285.

Lee ME, DeLoache WC, Cervantes B, Dueber JE. 2015. A Highly Characterized Yeast Toolkit for Modular, Multipart Assembly. ACS Synthetic Biology. doi:10.1021/sb500366v

Li W, Godzik A. 2006. Cd-hit: a fast program for clustering and comparing large sets of protein or nucleotide sequences. Bioinformatics 22:1658-1659.

Luo W-X, Cheng T, Guan B-Q, Li S-W, Miao J, Zhang J, Xia N-S. 2006. Variants of green fluorescent protein GFPxm. Mar Biotechnol 8:560-566.

Maynard Smith J. 1970. Natural selection and the concept of a protein space. Nature 225:563-564.

Melamed D, Young DL, Gamble CE, Miller CR, Fields S. 2013. Deep mutational scanning of an RRM domain of the Saccharomyces cerevisiae poly(A)-binding protein. RNA 19:1537-1551.

Milkman R. 1978. Selection differentials and selection coefficients. Genetics 88:391-403.

Murshudov GN, Vagin AA, Dodson EJ. 1997. Refinement of macromolecular structures by the maximum-likelihood method. Acta Crystallogr D Biol Crystallogr 53:240-255.

Nivón LG, Moretti R, Baker D. 2013. A Pareto-optimal refinement method for protein design scaffolds. PLoS One 8:e59004.

Ogden PJ, Kelsic ED, Sinai S, Church GM. 2019. Comprehensive AAV capsid fitness landscape reveals a viral gene and enables machine-guided design. Science 366:1139-1143.

Olson CA, Wu NC, Sun R. 2014. A comprehensive biophysical description of pairwise epistasis throughout an entire protein domain. Curr Biol 24:2643-2651.

Pédelacq J-D, Cabantous S, Tran T, Terwilliger TC, Waldo GS. 2006. Engineering and characterization of a superfolder green fluorescent protein. Nat Biotechnol 24:79-88.

Poelwijk FJ, Socolich M, Ranganathan R. 2019. Learning the pattern of epistasis linking genotype and phenotype in a protein. Nature Communications. doi:10.1038/s41467-019-12130-8

Pokusaeva VO, Usmanova DR, Putintseva EV, Espinar L, Sarkisyan KS, Mishin AS, Bogatyreva NS, Ivankov DN, Akopyan AV, Avvakumov SY, Povolotskaya IS, Filion GJ, Carey LB, Kondrashov FA. 2019. An experimental assay of the interactions of amino acids from orthologous sequences shaping a complex fitness landscape. PLoS Genet 15:e1008079.

Povolotskaya IS, Kondrashov FA. 2010. Sequence space and the ongoing expansion of the protein universe. Nature 465:922-926.

Renfrew PD, Choi EJ, Bonneau R, Kuhlman B. 2012. Incorporation of noncanonical amino acids into Rosetta and use in computational protein-peptide interface design. PLoS One 7:e32637.

Rocklin GJ, Chidyausiku TM, Goreshnik I, Ford A, Houliston S, Lemak A, Carter L, Ravichandran R, Mulligan VK, Chevalier A, Arrowsmith CH, Baker D. 2017. Global analysis of protein folding using massively parallel design, synthesis, and testing. Science 357:168-175.

Romero PA, Arnold FH. 2009. Exploring protein fitness landscapes by directed evolution. Nat Rev Mol Cell Biol 10:866-876.

Ronquist F, Teslenko M, van der Mark P, Ayres DL, Darling A, Höhna S, Larget B, Liu L, Suchard MA, Huelsenbeck JP. 2012. MrBayes 3.2: efficient Bayesian phylogenetic inference and model choice across a large model space. Syst Biol 61:539-542. 
Russ WP, Figliuzzi M, Stocker C, Barrat-Charlaix P, Socolich M, Kast P, Hilvert D, Monasson R, Cocco S, Weigt M, Ranganathan R. 2020. An evolution-based model for designing chorismate mutase enzymes. Science 369:440-445.

Sailer ZR, Shafik SH, Summers RL, Joule A, Patterson-Robert A, Martin RE, Harms MJ. 2020. Inferring a complete genotype-phenotype map from a small number of measured phenotypes. PLoS Comput Biol 16:e1008243.

Sardanyés J, Elena SF, Solé RV. 2008. Simple quasispecies models for the survival-of-the-flattest effect: The role of space. J Theor Biol 250:560-568.

Sarkisyan KS, Bolotin DA, Meer MV, Usmanova DR, Mishin AS, Sharonov GV, Ivankov DN, Bozhanova NG, Baranov MS, Soylemez O, Bogatyreva NS, Vlasov PK, Egorov ES, Logacheva MD, Kondrashov AS, Chudakov DM, Putintseva EV, Mamedov IZ, Tawfik DS, Lukyanov KA, Kondrashov FA. 2016. Local fitness landscape of the green fluorescent protein. Nature 533:397-401.

Senior AW, Evans R, Jumper J, Kirkpatrick J, Sifre L, Green T, Qin C, Žídek A, Nelson AWR, Bridgland A, Penedones H, Petersen S, Simonyan K, Crossan S, Kohli P, Jones DT, Silver D, Kavukcuoglu K, Hassabis D. 2020. Improved protein structure prediction using potentials from deep learning. Nature 577:706-710.

Srivastava N, Hinton G, Krizhevsky A, Sutskever I, Salakhutdinov R. 2014. Dropout: a simple way to prevent neural networks from overfitting. J Mach Learn Res 15:1929-1958.

Vagin A, Teplyakov A. 1997. MOLREP: an Automated Program for Molecular Replacement. J Appl Crystallogr 30:1022-1025.

Wagner A. 2008. Robustness and evolvability: a paradox resolved. Proc Biol Sci 275:91-100.

Weber E, Engler C, Gruetzner R, Werner S, Marillonnet S. 2011. A modular cloning system for standardized assembly of multigene constructs. PLoS One 6:e16765.

Wittmann BJ, Yue Y, Arnold FH. 2021. Informed training set design enables efficient machine learning-assisted directed protein evolution. Cell Syst. doi:10.1016/j.cels.2021.07.008

Wrenbeck EE, Faber MS, Whitehead TA. 2017. Deep sequencing methods for protein engineering and design. Current Opinion in Structural Biology. doi:10.1016/j.sbi.2016.11.001

Wright S. 1932. The roles of mutation, inbreeding, crossbreeding and selection in evolution. Proc Sixth Int Congr Genet 1:356-366.

Wu Z, Kan SBJ, Lewis RD, Wittmann BJ, Arnold FH. 2019. Machine learning-assisted directed protein evolution with combinatorial libraries. Proc Natl Acad Sci U S A 116:8852-8858.

Yang Z. 2007. PAML 4: phylogenetic analysis by maximum likelihood. Mol Biol Evol 24:1586-1591.

Zheng J, Guo N, Wagner A. 2020. Selection enhances protein evolvability by increasing mutational robustness and foldability. Science 370. doi:10.1126/science.abb5962

Zhou J, McCandlish DM. 2020. Minimum epistasis interpolation for sequence-function relationships. Nat Commun 11:1782. 